\title{
A Subjectivist's Guide to Deterministic Chance
}

\author{
J. Dmitri Gallow*
}

\begin{abstract}
I present an account of deterministic chance which builds upon the physico-mathematical approach to theorizing about deterministic chance known as the method of arbitrary functions. This approach promisingly yields deterministic probabilities which align with what we take the chances to be-it tells us that there is approximately a $\mathrm{I} / 2$ probability of a spun roulette wheel stopping on black, and approximately a I/2 probability of a flipped coin landing heads up-but it requires some probabilistic materials to work with. I contend that the right probabilistic materials are found in reasonable initial credence distributions. I note that, with some rather weak normative assumptions, the resulting account entails that deterministic chances obey a variant of LEWIs's 'principal principle'. I additionally argue that deterministic chances, so understood, are capable of explaining long-run frequencies.
\end{abstract}

\section{INTRODUCTION}

You have a better chance of winning at blackjack than at craps (even if you happen to win at neither). You have a better chance of getting into an accident at an intersection than at a roundabout (even if you happen to make it through the intersection unscathed and crash at the roundabout). A peacock with large and colorful feathers has a better chance of reproducing than a similar peacock with small and lackluster feathers (even if both of them end up finding a mate).

I take these truths to be evident. I am a compatibilist about chance. I believe that fundamental physical determinism is compatible with non-trivial chance. For instance, I believe that the outcome of a coin toss in a Newtonian universe - an outcome which is uniquely determined by the initial conditions of the tossed coin-is a matter of chance. According to compatibilists, ${ }^{\mathrm{I}}$ the chance that this coin lands heads is approximately $50 \% .^{2}$ According to incompatiblists, ${ }^{3}$

Final Draft. Forthcoming in Synthese.

* Thanks to Michael Caie, Cian Dorr, Daniel Drucker, Jeremy Goodman, Zoë Johnson King, Harvey Lederman, Jonathan Livengood, Japa Pallikkathayil, Bernhard Salow, James Shaw, Erica Shumener, Charles Sebens, Jack Spencer, Michael Strevens, Rohan Sud, Brad Weslake, two anonymous reviewers, and the Logic, Language, Metaphysics, and Mind Reading Group at MIT for helpful conversations about this material.

I For instance, Clark (1987) Loewer (200I), Hoefer (2007), Ismael (2009), Glynn (20io), Sober (2OIO), and Strevens (2OII).

2 Strictly speaking, a compatibilist needn't say this. They could say that the chance is $2 / 3$, or $\pi / 4$, or even o or I. All it takes to be a compatibilist is to assign some proposition a non-trivial chance in a deterministic world.

3 For instance, Popper (I982), Lewis (I986), and Schaffer (2007). 
the chance that the coin lands heads is I, if it actually does, and o, if it actually doesn't.

An incompatibilist will disagree with me about whether chance is compatible with fundamental physical determinism, yet they are likely to accept the claims in my first paragraph—or at least some suitably sterilized translation of those claims. Perhaps they will say that roundabouts decrease the epistemic probability, or what-have-you, of accidents, while still insisting that the chance of an accident, in a deterministic world, is $\mathrm{I}$, if the accident occurs, and o, if the accident doesn't occur. ${ }^{4}$ What is to keep our disagreement from being merely verbal? Perhaps nothing. Perhaps, once 'chance' has been exchanged for 'epistemic probability', or what-have-you, the incompatibilist will accept all the same claims about chance as I. Such an incompatibilist and I have different views about how the word 'chance' ought to be used; but we don't disagree at all about the nature of chance (or, if you are such an incompatibilist: we don't disagree at all about the nature of epistemic probability). 5

For my part, I do think that the word 'chance' is appropriately used to describe deterministic systems; so, when I am speaking, I will use the word 'chance' indiscriminately, to refer both to the kinds of chances that exist in worlds whose fundamental physical laws are deterministic and the kinds of chances that are unique to worlds whose fundamental physical laws are indeterministic. The former variety of chance I refer to as 'deterministic chance'. The latter variety I call 'tychistic chance.' Since my goal is not to argue for any thesis about the meaning of 'chance', you should feel free to translate into your own idiom as you see fit. Nevertheless, even after such translation, disagreement about the nature of deterministic chance may remain.

To see why, we should turn to the sorts of considerations that motivate incompatibilism in the first place. I take it that one of the central intuitions motivating incompatibilism is well illustrated by the coin-tossing machine constructed by Diaconis et al. (2007). This machine can exercise such minute control over the initial conditions of the toss that it can determine whether the coin lands heads or tails. If the coin is placed in the machine heads-up, then it will land heads-up; if it is placed in the machine tails-up, then it will land tails-up. ${ }^{6}$ It is incredi-

4 See, for instance, Schaffer (2007).

5 Following philosophical tradition, I reserve the word 'chance' for objective probabilities. So, when I call the probabilistic features of deterministic systems 'chances', I mean to place those probabilities on the objective side of an objective-subjective dichotomy. It may be that the reader disagrees with me about whether there are deterministic chances because they and I disagree about where to draw the line between objective and subjective. This is not first and foremost a disagreement about the nature of the probabilistic features of deterministic systems. It is rather first and foremost a disagreement about how to use our terms. Others, like Schaffer (2007) and Bradley (20I7), will think that chance is whatever plays (well enough) certain theoretical roles like constraining rational credence and explaining frequencies. Part of my goal here is to demonstrate that the probabilistic features ascribed to deterministic systems are capable of playing these kinds of theoretical roles.

6 I take the existence of this machine to decisively settle, in the negative, LEWIS (I986, p. II9)'s question of whether quantum mechanical chance will infect the tossing of a coin to a degree 
bly difficult to see the outcome of this machine's coin toss as a matter of chance. In the presence of such a machine, one feels as though there simply isn't anything chancy about the outcome of the machine's coin toss at all—or, perhaps, one feels that, when the coin is placed in the machine heads up, the chance that the coin lands heads is I (or as near as tychistic chance will allow-which is very near). Moreover, there doesn't appear to be any important difference between the machine's coin tosses and our coin tosses-other than, of course, our epistemic situation with respect to the outcome. In the case of the machine, we know how the coin will land. In the case of our coin toss, we don't. It may well be that the initial conditions of our tossed coin are exactly the same as the initial conditions of the machine's tossed coin. So it is quite tempting to conclude that deterministic chance is just a matter of our epistemic situation with respect to the outcome, that deterministic chance is not a worldly affair. Thus LAPLACE (I8I4) writes:

The curve described by a simple molecule of air or vapor is regulated in a manner just as certain as the planetary orbits; the only difference between them is that which comes from our ignorance... Probability is relative, in part to this ignorance, in part to our knowledge. ${ }^{7}$

But wait-deterministic chances don't just earn their keep by telling us how confident to be in the outcome of deterministically chancy processes. They additionally help to explain certain worldly facts, such as the frequency of heads in a long series of trials. But facts about our epistemic situation aren't capable of explaining the outcome of a physical process like a coin toss. Moreover, chances appear to be sensitive to physical features of the world, even when we are ignorant of those features. A casino could hardly protest the charge of using an unfair die by pointing out that nobody knew that the die was loaded. ${ }^{8}$ Whether the die is fair seems to be entirely a question of how the mass in the die is distributed, and not at all a question of our, or anybody's, epistemic situation with respect to the distribution of mass in the die.

Deterministic chance is an odd sort of beast. It appears at once subjective and epistemic, a chimera of our ignorance, and objective and worldly, the kind of thing that depends upon and helps to explain features of the natural world. It is my view that an adequate account of deterministic chance should help to explain this dual nature. My goal here is to provide such an account. So put aside the question of whether the probabilistic features ascribed to deterministic systems are appropriately called 'chances'. What's interesting in this question-what remains after merely verbal disagreement is settled-is whether what I choose to call 'deterministic chance' (whatever you choose to call it) is a chimera of our ignorance or a worldly affair. My answer will be: in some ways, deterministic chance is a matter of our ignorance; in some ways, it is a worldly affair-the devil, of course,

sufficient to render the tychistic chance of heads I/2.

7 Laplace (I8I4, p. 6)

8 When I say that the die is 'unfair', I mean that it is not the case that the chance that one side land up is the same as the chance that any other side land up. 
is in the details. To the extent that incompatibilists deny the worldly aspects of deterministic chance, they and I disagree. Equally, to the extent that compatibilists reject the epistemic aspects of deterministic chance, they and I disagree.

My account of deterministic chance will take, as its jumping-off point, a physico-mathematical approach to theorizing about deterministic chance known as the method of arbitrary functions. This approach is motivated by the insight that deterministic systems like the spinning of a roulette wheel or the tossing of a coin in a Newtonian universe have an interesting property: almost any reasonable probability distribution over the initial conditions of the roulette wheel's spin or the coin's toss will induce a probability very close to 50\% to the roulette wheel landing on black and the coin's landing heads $(\$ 2)$. It is tempting to try to parlay this physico-mathematical result into a metaphysical account of deterministic chance. Any account along these lines faces the obstacle of explaining away the probability distributions over initial conditions (call these the initial distributions). For if the initial distributions are chance distributions, then we have not analyzed away the notion of chance, nor in any way illuminated its compatibility with determinism. We have, rather, merely said something about how the world's causal structure and its chance function are interrelated. Some have attempted to parlay the method of arbitrary functions into an account of deterministic chance by claiming, roughly, that the initial distribution is just a measure of the proportion of those initial conditions. ${ }^{9}$ Others claim that the initial distributions come from the actual and/or hypothetical frequencies of initial conditions, ${ }^{\text {IO }}$ or that the initial distribution is itself a law of nature. II In contrast, I will suggest that, roughly, these initial distributions are reasonable prior credal distributions over the initial conditions $\left(\$_{4}\right) .^{\mathrm{I} 2}$ I will argue that this approach is able to explain why chances play the role they do in scientific practice and every day reasoning. In particular, I will argue, it is able to show how chances are capable of explaining long-run frequencies and how they are capable of constraining rational credence. That's because the account on offer here, together with some plausible theses about rational credence, ends up entailing a deterministic variant of LEwIs's 'principal principle', which tells us, roughly, that our credence in a proposition $p$, conditional on the chance of $p$ being $x$, ought to be $x$.

\section{The Method of Arbitrary Functions}

In his Calcul des Probabilités ( $\$ \$ 92 \& 93$ ), PoInCARÉ introduced a method for determining the chancy properties of deterministic systems which has come to be known as the method of arbitrary functions. ${ }^{13}$ In this section, I will introduce the

9 See Rosenthal (20IO, 20I2, 20I6).

io See Strevens (2003, 20II), Abrams (20I2), and Beisbart (20I6).

II See Albert (2000, 20I5), Loewer (200I, 2004, 2007), and Roberts (2016).

I2 This puts me in the company of SAVAGE (197I) and Myrvold (20I2).

I3 VON KRIES (I886) scooped PoinCARé by several years, though he left the mathematical details rather vague. See von Plato (1982) and Zabell (2016). 
method of arbitrary functions by way of two examples, and then say something about how to generalize the approach. ${ }^{\mathrm{I}}$

\section{I The Roulette Wheel}

For purposes of illustration, consider the deterministic system of a croupier's spinning a roulette wheel. Just to keep things simple, let's suppose that the roulette wheel consists of 36 equally sized, colored pockets. The color of the pockets alternates between black and red, so that half of the pockets are colored black, and half are colored red. There is a fixed pointer, and the color of whichever pocket it points to when the wheel is finished spinning is the winning color. Suppose that the pointer begins pointing at the start of a black pocket, the croupier gives the wheel an initial angular velocity of $V$, and friction causes the wheel's angular velocity to decelerate at a constant rate, $\delta$. Then, the wheel's angular velocity at $t$ will be $V-\delta t$. It will therefore stop rotating at time $t^{*}=V / \delta$. At time $t$, the wheel will have rotated $\theta_{t}$ degrees, where $\theta_{t}=V t-0.5 \delta t^{2}$. Thus, at the time $t^{*}$ when the wheel stops rotating, it will have rotated a total number of degrees $\theta_{t^{*}}$, where $\theta_{t^{*}}=V t^{*}-0.5 \delta t^{* 2}=V^{2} / 2 \delta$. Thus, if $V^{2} / 2 \delta$ is between $0^{\circ}$ and $10^{\circ}$, then black will win; if $V^{2} / 2 \delta$ is between $10^{\circ}$ and $20^{\circ}$, then red will win; if $V^{2} / 2 \delta$ is between $20^{\circ}$ and $30^{\circ}$, then black will win; and so on.

This is illustrated in figure $\mathrm{I}$, where the area above a velocity, $V$, is shaded if a spin with that initial velocity would lead to the roulette wheel stopping on a black pocket, and is left unshaded if a spin with that initial velocity would lead to the roulette wheel stopping on a red pocket. Notice that, as the initial velocity $V$ gets greater, the distance between shaded and unshaded regions gets smaller. In the limit as $V$ goes to infinity, this distance converges towards zero. (This is because the number of degrees the wheel rotates is a function of $V^{2}$.)

The basic thought behind the method of arbitrary functions is just this: we may pick any probability density function over the croupier's spin velocity $V$ (call this an initial distribution), and this will induce a probability very close to one half to black's winning, and a probability very close to one half to red's winning. That is, we may pick an arbitrary initial distribution, and we will arrive at a probability of very nearly one half for black and a probability of very nearly one half for red. See figures 2-4. That is the thought; but a moment's reflection shows that it can't be quite right. It's not the case that any initial distribution will induce a probability very close to one half to black's winning. Consider the initial distribution shown in figure 5. This initial distribution will induce a probability much greater than one half to the roulette wheel landing on black, and a probability much less than one half to the roulette wheel landing on red. (Call this 'the problem of all too

I4 A note on the presentation: the method of arbitrary functions has a long and storied history. My goal in this section is not to give anything like an adequate introduction to the historical development of these ideas, but rather to simply present them in the form that is currently fashionable. See Strevens (2003, \$2.A) and von Plato (1983) for accessible introductions to this historical development. See ENGEL (1992) for a more technical historical introduction. 


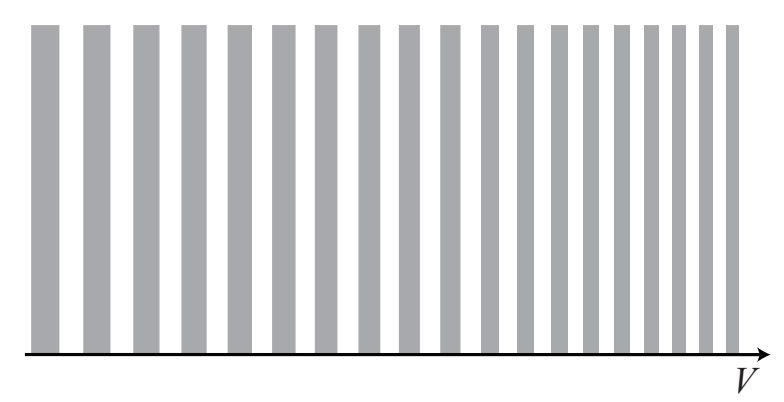

FIGURE I: The outcome of the roulette wheel spin, as a function of its initial angular velocity. If the initial angular velocity is in a region shaded grey, then the wheel will stop on black; else, it will stop on red.

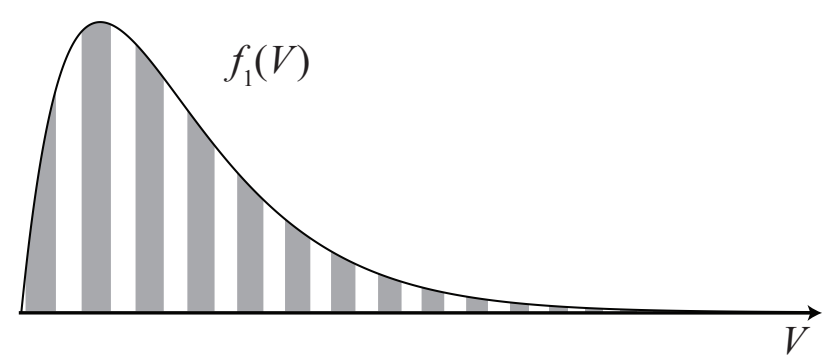

Figure 2: The area under the curve $f_{1}$ mapped to black, $\int_{\mathbf{B}} f_{1}(V) d V \approx \mathrm{I} / 2$.

arbitrary functions'.)

A standard way of getting around the problem of all-too arbitrary functions is to shift attention towards various asymptotic features of the analysis. For instance, PoINCARÉ (I9I2) points out that, as the number of equally-sized red and black pockets on the roulette wheel goes to infinity, the probability of black goes to $\mathrm{I} / 2$, given any absolutely continuous initial distribution-even the one shown in figure 5. Hopf (1934) similarly points out that, given any absolutely continuous initial distribution, as the friction on the roulette wheel goes towards zero, the probability of the wheel's stopping on black approaches $\mathrm{I} / 2$. Others observe that, so long as the initial distribution is absolutely continuous, in the limit as the initial angular velocity $V$ goes to infinity, the probability of the wheel's stopping on black approaches $\mathrm{I} / 2^{15}$ - this is because, as $V$ gets larger, the gaps between initial velocities leading to black and initial velocities leading to red get smaller. ${ }^{16}$

These are interesting observations, but it isn't clear what relation these asymp-

is That is to say: $\lim _{x \rightarrow \infty} \int_{\mathbf{B}} f(V-x) d V=\mathrm{I} / 2$.

I6 See Keller (1986), in which he determines the probability of heads as the initial upwards and angular velocities go to infinity, supposing that the initial distribution is absolutely continuous. 


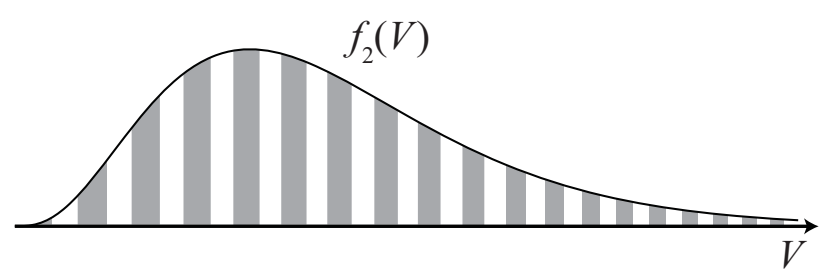

Figure 3: The area under the curve $f_{2}$ mapped to black, $\int_{\mathbf{B}} f_{2}(V) d V \approx \mathrm{I} / 2$.

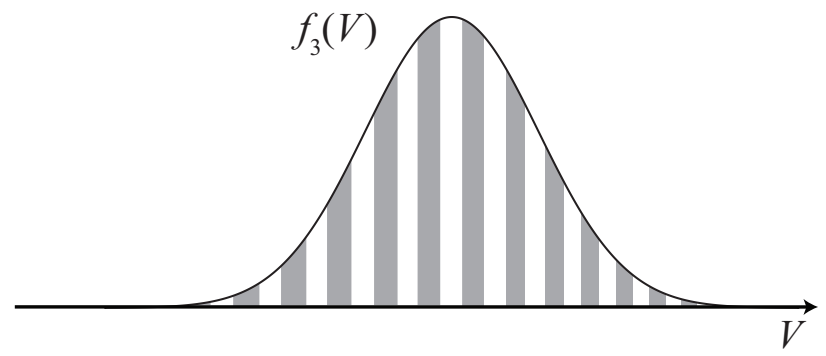

Figure 4: The area under the curve $f_{3}$ mapped to black, $\int_{\mathbf{B}} f_{3}(V) d V \approx \mathrm{I} / 2$.

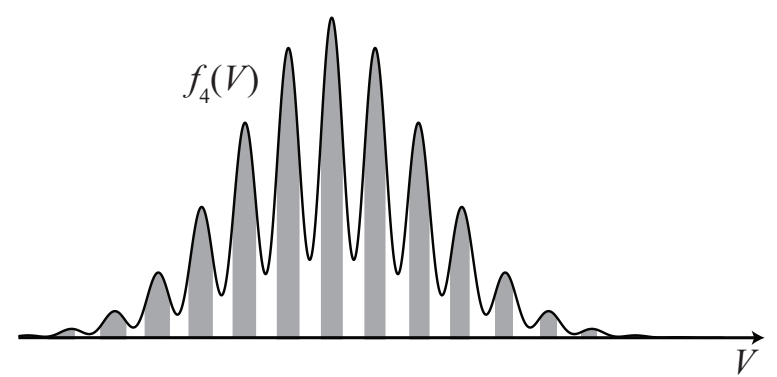

Figure 5: The problem of all-too arbitrary functions. Not just any probability distribution will give a probability of $\mathrm{I} / 2$ to the set of initial conditions which lead to the roulette wheel stopping on black. $\int_{\mathbf{B}} f_{4}(V) d V \gg \mathrm{I} / 2$. 
totic results are supposed to bear to the chance of black. Consider the final approach: even putting aside the worry that the laws of nature put an upper bound on the value of $V$, the dynamical equation that we were relying upon when we assumed that the initial velocity determines the outcome of the spin simply does not hold when the velocity is too large. Were the initial velocity $V$ to get close to the speed of light, the air molecules wouldn't have time to get out of the way of the spinning roulette wheel. Those molecules would fuse with the atoms in the surface of the wheel, releasing a massive amount of energy. There simply wouldn't be an answer to the question of whether the pointer points at a black or red pocket, as both the wheel and the pointer would be almost instantly vaporized by the ensuing thermonuclear reaction. The point here is just that the method of arbitrary functions is relying upon certain dynamical equations which we ought to trust in the case of low velocities, but which we have positive reason to distrust in the case of high velocities.

Even if we don't find this kind of response troubling - perhaps because we think that limiting behavior can be revelatory, even when we know that that limit is in principle unreachable ${ }^{\mathrm{I} 7}$ - relying upon limiting probabilities to determine chances makes it unclear what relationship there could be between the chance of the roulette wheel stopping on black and the observed frequency of roulette spins which stop on black. For we know that all of the actual initial velocities are quite low; why, then, would we expect to learn anything about the actual frequencies by looking at a probability distribution most of whose mass is atop astronomically high velocities? ${ }^{18}$ In sum, it doesn't seem to me that the appeal to asymptotics is an effective response to the problem of all-too-arbitrary functions. I'll put it aside from here on out. But this leaves an outstanding problem that an adequate account of deterministic chance built upon the method of arbitrary functions must solve.

\subsection{The CoIn Flip}

The method of arbitrary functions analysis is meant to apply to more systems than a croupier's spin of a roulette wheel. For another example of this reasoning at work, consider KeLLER (I986)'s analysis of the probability that a flipped coin will land heads-up. KeLLER asks us to imagine that we flip a coin with radius $r$, initially laying flat at height $r$ above the ground with its tails face up, ${ }^{19}$ by providing it with a certain initial upward velocity $U$ and a certain angular velocity $A$ (see figure 6). Suppose that the coin rotates about its center of mass with a constant angular velocity. Since, in the earth's gravitational field, the vertical acceleration of the coin is $-g$, the coin's height at time $t$ is $h_{t}=U t-0.5 g t^{2}+r$. If we assume that the coin does not bounce, then whatever side of the coin is facing up

17 See Strevens (2003, \$2.A) and Butterfield (20II).

I8 See Strevens $\left(2011, \$_{3}\right)$

I9 Following Keller, I set the coin's initial height equal to its radius in order to simplify the math. Nothing significant changes if we vary the coin's initial height. 

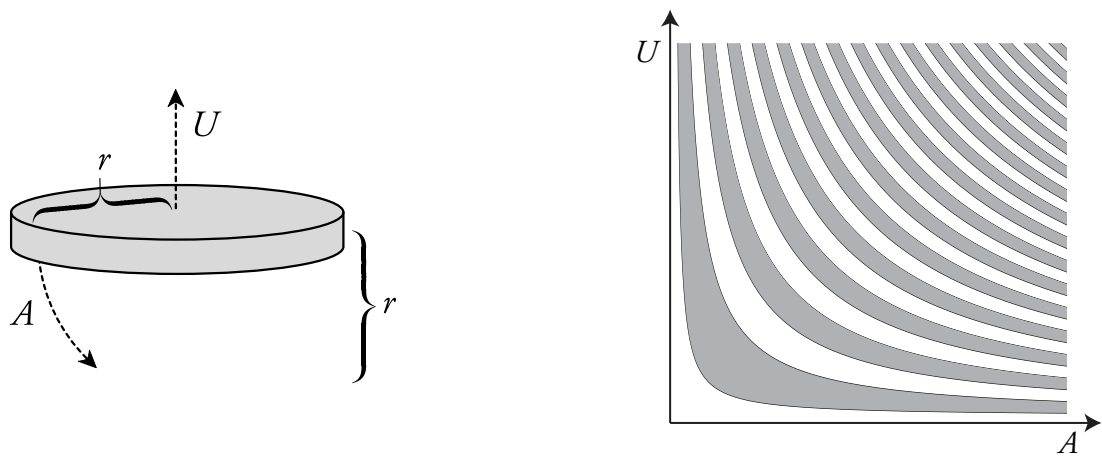

FIgURE 6: The outcome of a coin flip as a function of its initial upward velocity $(U)$ and its initial angular velocity $(A)$. If $\langle U, A\rangle$ lies in the grey area, then the coin will land heads; otherwise, it will land tails.

when it returns to the height $r$ will be the side which is facing up after the coin has settled to the ground. The coin will return to height $r$ at time $t^{*}=2 \mathrm{U} / \mathrm{g}$. Because the coin's rate of rotation $A$ is constant, the angle the coin has rotated at time $t, \theta_{t}$, will be equal to $A t$. Thus, when the coin returns to height $r$, it will have rotated $\theta_{t^{*}}=2 A U / g$ degrees. Since the coin began tails up, if $2 A U / g$ is between $90^{\circ}$ and $270^{\circ}$, then coin will land heads. If $2 A U / g$ is between $270^{\circ}$ and $450^{\circ}$, then the coin will land tails. If $2 A U / g$ is between $450^{\circ}$ and $630^{\circ}$, then the coin will land heads; and so on.

If we graph those pairs of initial upward velocities and initial angular velocities $\langle U, A\rangle$ which lead to the coin landing heads, we get the hyperbolas shown in figure 6. As in the case of the roulette wheel, just about any not-too-arbitrary probability distribution over the values of $\langle U, A\rangle$ will put about half of its mass on values of $\langle U, A\rangle$ which lead to the coin landing heads and about half of its mass on values of $\langle U, A\rangle$ which lead to the coin landing tails. ${ }^{20}$

\subsection{The Method of Arbitrary Functions in General}

Suppose that there is a variable $O$ (which we can think of as the outcome of the chance process) such that the value of $O$ is causally determined by the values of some other set of variables $C_{1} \ldots C_{N}$ (which we can think of as the causes of the outcome $O$ ). I'll assume that what it is for $O$ to be causally determined, in the relevant way, by the values of $C_{1} \ldots C_{N}$, is for there to be a true dynamical equation of the form $O:=\phi_{O}\left(C_{1}, \ldots, C_{N}\right){ }^{2 \text { I }}$ In the case of the roulette wheel, we had the function $B:=\phi_{B}(V)$, where $V$ is the initial angular velocity of the roulette wheel, $B$ is a binary variable which takes the value 1 if the roulette wheel stops on black,

20 For a discussion of the application of the method of arbitrary functions to several other chance processes, see Engel (1992) and StreVens (2003, 2013). For a development of Keller's analysis which incorporates precession and shows surprisingly that a flipped coin is slightly more likely to land on whichever face was up initially $(\approx 5 \mathrm{I} \%)$, see DiAcONIS et al. (2007).

2I I'm using ':=', rather than '=', to emphasize that the value of $O$ is determined by the values of $C_{1} \ldots C_{N}$, and not vice vers.$:=$ is therefore, not a symmetric relation like $=$. 
and takes the value 0 if it instead stops on red, and $\phi_{B}$ is ${ }^{22}$

$$
\phi_{B}(V)= \begin{cases}1 & \text { if } V^{2} / 2 \delta \bmod 20<10 \\ 0 & \text { if } V^{2} / 2 \delta \bmod 20 \geqslant 10\end{cases}
$$

(This is the function illustrated in figure $\mathrm{I}$ ). In the case of the coin flip, we have the function $H:=\phi_{H}(U, A)$, where $U$ is the coin's initial upward velocity, $A$ is the coin's initial angular velocity, $H$ is a binary variable which takes the value 1 if the coin lands heads and takes the value 0 if it instead lands on tails, and $\phi_{H}$ is

$$
\phi_{H}(U, A)=\left\{\begin{array}{lll}
1 & \text { if } & (2 U A / g-90) \bmod 360<180 \\
0 & \text { if } & (2 U A / g-90) \bmod 360 \geqslant 180
\end{array}\right.
$$

(This is the function shown in figure 6.)

A method of arbitrary functions analysis of the deterministic chance of a certain outcome $O=o$ consists of two components: a dynamical equation $\phi_{O}$ from $C_{1} \ldots C_{N}$ to $O$ and an initial probability distribution $f$ over the potential values of $C_{1} \ldots C_{N} \cdot{ }^{23}$ From the initial distribution over the values of $C_{1} \ldots C_{N}$, we induce a probability distribution over the values of $O$ as follows: we look to the set of initial conditions such that, according to the dynamical equation $\phi_{O}$, those initial conditions lead to the outcome $O=o$. Denote this set of initial conditions with ' $\phi_{O}^{-1}[O=o]$ '. Then, we set the probability of $O=o$ equal to the probability of its causes, $\phi_{O}^{-1}[O=o]$.

$$
f(O=o)=f\left(\phi_{O}^{-1}[O=o]\right)
$$

Because it gives us a relation between a probability function and a dynamic equation, I call this equation the 'probability dynamics principle' (or 'PDP'). The PDP says, of any given probability function, $f$, that the world's causal dynamics take $f$ along for the ride. The PDP is non-trivial. There are many probability functions for which it obviously does not hold-for instance, if $f$ is an individual's credence function, ${ }^{24}$ and that individual is ignorant of the causal relationship between $C_{1} \ldots C_{N}$ and $O$, then there is no reason to expect that PDP will hold of $f$. However, it seems very plausible as a constraint on the relationship between chance and causation. Indeed, many authors who rely upon the PDP fail to explicitly mention the principle at all. Those who do mention the principle usually do so without comment.

As a first pass, then, the method of arbitrary functions (MAF) analysis of de-

22 ' $a \bmod b$ ' is the remainder when $a$ is divided by $b$.

23 A few words on notation. Throughout, I will use ' $\phi_{O}$ ' to stand for both the function on the righthand-side of a dynamical equation $O:=\phi_{O}\left(C_{1}, C_{2}, \ldots, C_{N}\right)$, and also the entire dynamical equation itself. Also, I will use ' $f$ ' indiscriminately to denote both a probability density function (if the variables over which it is defined are continuous), a probability mass function (if the variables over which it is defined are discrete), and the probability function determined by such pdfs or pmfs.

24 I will be assuming throughout that rational credences are probabilities. 
terministic chance says the following:

METHOD OF ARBITRARY FUNCTIONS (MAF)

If $\phi_{O}$ and $f$ are suitable, then

$$
C h(O=o)=f\left(\phi_{O}^{-1}[O=o]\right)
$$

That is: the chance distribution over outcomes is the result of time-evolving a suitable probability distribution along a suitable dynamical equation $\phi_{O}$, according to the probability dynamics principle, PDP. In a slogan: chance is a suitable probability distribution filtered through suitable dynamics. Once a suitable probability distribution is supplied, the MAF will tell us that a fair coin has about a half chance of landing heads and that a fair roulette wheel has about a half chance of stopping on black. So too will it tell us that, if a die has most of its mass concentrated opposite its 6-face, then it will have a greater than one-sixth chance of landing with its 6 face up; and that, if the roulette wheel has a greater coefficient of friction in its black regions than its red regions, then it will have a greater than one half chance of stopping on black.

The restriction to suitable $\phi_{O}$ and $f$ in MAF is important. Not just any initial distribution $f$ will do. As we saw in $\$ 2.1$, if we allow just any initial distribution, then, by appeal to gerrymandered distributions like the one shown in figure 5, we could give $O=O$ any chance we like. Similarly, not just any dynamical equation $\phi_{O}$ will do. At the least, this equation must be correct. Perhaps more than correctness is required for a dynamical equation to be suitable for the MAF analysis. STREVENS (2003, 20II, 2013) places a joint requirement on both the initial distribution $f$ and the dynamical equation $\phi_{O}$ : he requires, firstly, that the dynamics be such that, for any small (but not too small) region of the initial conditions space $\left\langle C_{1}, \ldots, C_{N}\right\rangle$, the proportion of initial conditions which lead to the outcome $O=o$ is about the same. ${ }^{25}$ If $\phi_{O}$ meets this requirement, Strevens says that it is microconstant. In the definition of microconstancy, how small is small enough (and how small is too small) is left vague. Given a certain standard for smallness, however, Strevens requires that the initial distribution $f$ be approximately uniform over small regions of the initial conditions space. STREvENS calls an initial distribution satisfying this condition macroperiodic. On Strevens' view, microconstancy and macroperiodicity are necessary conditions on $\phi_{O}$ and $f$ being suitable. Notice that these conditions will, all by themselves, rule out all-too-arbitrary functions like the one shown in figure 5. Such functions are not macroperiodic because they are not approximately uniform over the small (but not too small) regions relative to which the dynamics are microconstant.

It is unclear whether microconstancy and macroperiodicity should be taken

${ }_{25}$ As I will be understanding the term 'microconstant', the proportion here is calculated with the Lebesgue measure, corresponding to the intuitive length of a set of points in $\mathbb{R}$, the intuitive area of a set of points in $\mathbb{R}^{2}$, the intuitive volume of a set of points in $\mathbb{R}^{3}$, etc. For a more careful definition of Lebesgue measure, see BARTLE (1966). 
to be necessary for the existence of a deterministic chance. ${ }^{26}$ It seems likely that microconstancy and macroperiodicity are necessary conditions for the existence of a deterministic chance whose value is (near enough) determinate. For nonmicroconstant dynamics and non-macroperiodic initial distributions, it may be that the most that is determinately true about the chance of the outcome is that it lies in some rather wide interval; but there's no obvious reason why this should preclude us from saying, for instance, that the chance is non-zero or that some event would raise or lower the chance of the outcome.

\section{The Method of Arbitrary Functions and the Metaphysics of Deterministic Chance}

The physico-mathematical results from the literature on the method of arbitrary functions are just that: mathematics and physics. These results on their own do not establish any thesis about the possibility or the nature of deterministic chance. Whether these results are capable of founding a metaphysics of deterministic chance depends upon how we unpack the 'suitability' requirement from MAF. In particular, until more is said about the suitability of $f$, there is nothing in the result from $\$ 2.2$ even guaranteeing that, in a deterministic universe, the chance of a coin landing heads is strictly between o and I. A staunch incompatiblist could affirm MAF but insist that the only suitable initial distribution is an initial chance distribution. And since non-trivial chance is ruled out by determinism, in deterministic worlds, $f$ would be a probability measure which places all of its mass on the actual initial conditions and none of its mass on any non-actual initial conditions.

In addition to a general skepticism about the possibility of deterministic chance, there are at least two reasons to be sympathetic to this kind of reaction. In the first place, there is a technical objection. ${ }^{27}$ Given any collection of variables, $C_{1}, \ldots, C_{N}$, which causally determine the value of $O$ in the manner specified by the equation $\phi_{O}$, we can cook up another collection of variables, $\zeta\left(C_{1}, \ldots, C_{N}\right)$, which are transformations of $C_{1}, \ldots, C_{N}$, and which determine the value of $O$ in a manner specified by a different dynamical equation $\zeta\left(\phi_{O}\right)$. And though these transformations will be quite unnatural and gerrymandered, we may cook up these alternative variables so as to make the proportion of the transformed initial conditions which lead to the outcome $O=o$ take on any value we like. ${ }^{28}$ In applying MAF, we insisted that the initial distribution over the initial condition space $\left\langle C_{1}, \ldots, C_{N}\right\rangle$ be not too arbitrary-i.e., that it be macroperiodic. The skeptic objects: why do we only require the initial distribution to be macroperiodic over that initial condition space? For after all, a macroperiodic initial distribution

${ }_{26}$ Of course, STREVENS need not, and does not, claim that they are. MAF provides a sufficient, and not a necessary, condition for the existence of a deterministic chance.

27 The objection is discussed in (STREVENS, 2003, chapter 2), and further emphasized by RosenTHAL (2OI2).

28 The proportion here is calculated with the Lebesgue measure. See footnote 25 . 
over $\left\langle C_{1}, \ldots, C_{N}\right\rangle$ may correspond to a non-macroperiodic distribution over the transformed initial conditions space.

I believe that there is a problem here. However, it is not, first and foremost, a problem for an account of deterministic chance built upon MAF. After all, it is important to the MAF analysis that the dynamical equation $\phi_{O}$ be correct. And it is generally thought that only certain natural variables are fit to enter into true dynamical equations of this kind. There is, of course, work to be done in explaining the eligibility of certain variables and the ineligibility of others. ${ }^{29}$ But this work is properly viewed as prior to the work of providing an account of deterministic chance in terms of dynamical equations such as these. So I'll put aside worries about gerrymandered transformations of variables from here on out and assume that correct dynamical equations only utilize the kinds of standard variables we are familiar with—position, velocity, mass, unemployment, inflation, and so on-or not-too-unnatural transformations of them (e.g., the kind of transformations involved in unit-conversion). ${ }^{30}$

Rosenthal (20I2, p. 23I) raises what is, I believe, a deeper objection to the project of using the MAF analysis to underpin an account of deterministic chance. The objection focuses on cases in which there are natural, salient, and microconstant dynamical equations $\phi_{C_{i}}$ describing how the values of $O$ 's causes, $C_{1}, \ldots, C_{N}$, are determined by the values of some prior variables, $A_{1}, \ldots, A_{M}$. In those cases, we may compose the functions $\phi_{O}$ and $\phi_{C_{i}}$ to get a dynamical equation $\phi_{O} \circ \phi_{C_{i}}$ which maps values of $A_{1}, \ldots, A_{M}$ to values of $O$. That is: once we know both how the variables $A_{i}$ determine the variables $C_{i}$ and how the variables $C_{i}$ determine the variable $O$, we have all we need to know how the variables $A_{i}$ determine the variable $O$. And there is no reason to expect a macroperiodic distribution over the values of $A_{1}, \ldots, A_{M}$ and a macroperiodic distribution over the values of $C_{1}, \ldots, C_{N}$ to assign the same probability to the outcome $O=o$. Thus, there may be multiple deterministic chances of the outcome $O=o$.

For a concrete example of a case like this, consider SLOT MACHINE.

\section{SLOT MACHINE}

Suppose that this is how a casino's slot machine works: it has a pseudo-random number generator which produces a real number between $O$ and $\mathrm{I}$ on each pull of the lever. If a lever pull produces a number in a certain designated range - say, within the interval $[0.222,0.223]$ - then that lever pull gets a payout. Here's how the pseudo-random number generator works: it takes a given number

29 See, for instance, Woodward (2016).

30 From the perspective of fundamental physics, the variables used to describe many social and biological systems will appear quite unnatural. Might we then expect some proportion-altering transformation $\zeta$ to deliver variables just as natural as $C_{1}, \ldots, C_{N}$ themselves? Perhaps, though I'm inclined to think not; for I don't think that the naturalness of high-level variables is to be judged by reducing such variables to the quantities of fundamental physics. So I'm inclined to think that the standard variables used in higher-level sciences are themselves rather natural, and the proportion-altering transformations of them rather unnatural. 
between o and I, $S_{0}$, known as the seed value, and then determines the value of the next number, $S_{1}$, which corresponds to the first pull of the lever, according to the recurrence equation $S_{1}=\left(a S_{0}+b\right)$ $\bmod 1$, for some $a, b>0$. The next value, $S_{2}$, corresponding to the second pull of the lever, is then determined by $S_{2}=\left(a S_{1}+b\right) \bmod 1$. In general, $S_{n+1}=\left(a S_{n}+b\right) \bmod 1$, for $n \geqslant 0$. Thus, given the initial seed value and the recurrence equation, it is completely determined whether the slot machine will pay out on the 3ooth pull. Suppose additionally that the initial seed value, $S_{0}$, was picked in the following manner: the casino owners took six randomly selected potential seeds, $R_{1}, \ldots, R_{6}$, and then rolled a standard die to determine which one to feed into the slot machine's recurrence equation. When the casino owners did this, of the six potential seeds, there was one (and only one), $R_{3}$, which would lead to the slot machine paying out on the 300 th pull.

In the scenario described in SLOT MACHINE, we could write down a true dynamical equation according to which whether the slot machine pays out on the 30oth pull—denote this binary variable with ' $P$ ', for 'payout' - is determined by the initial seed value, $S_{0}$. This equation will tell us that the machine will pay out on the 300 th pull just in case the initial seed value lies within a range of [o, I] that gets mapped to $[0.222,0.223]$ by 300 applications of the recurrence equation. Call this equation ' $\phi_{P}$ '. And we could write down another true dynamical equation according to which the initial seed value is determined by the initial conditions of the die roll-if those initial conditions lead to the die landing with its $i$ face up, then $R_{i}$ is the initial seed. Call this equation $\phi_{S}$-where ' $S$ ' is for 'seed'. But then, by composing $\phi_{P}$ and $\phi_{S}$, we get an equation according to which whether the slot machine pays out on the 300 th pull is determined by the initial conditions of the die roll. According to this equation $-\phi_{P} \circ \phi_{S}$ - the machine will pay out on the 3ooth pull just in case the initial conditions of the die roll lie within a range that lead to the die landing with 3 face-up. Assuming that the values of $a$ and $b$ are suitable, both $\phi_{P}$ and $\phi_{P} \circ \phi_{S}$ will be microconstant, in STREVEns' sense. Let's also suppose that we've chosen our values of $a$ and $b$ so that a macroperiodic probability distribution over the initial seed value, $S$, will assign probability of approximately I/Iooo to the machine paying out on the 30oth pull—and a macroperiodic distribution over the initial conditions of the die roll will assign a probability of about $\mathrm{I} / 6$ to the machine paying out on the 300 th pull. ${ }^{3 \mathrm{I}}$

3I A function like $\phi_{P}$ will only be microconstant if we choose an appropriate $a$ and $b$. To persuade yourself that this will work out for some choice of $a$ and $b$, I recommend playing around in Mathematica. For instance, to get a sense of how functions like this behave, you can set $a=9245.8698$ and $b=6.282$. Then, define the function $\mathrm{f}\left[\mathrm{x}_{-}\right]:=\operatorname{Mod}[\mathrm{a} * \mathrm{x}+$ $\mathrm{b}, 1]$, which says what $S_{n+1}$ will be, given the input $S_{n}$. To see what this function produces when iterated 300 times, define $g\left[x_{-}\right]:=\operatorname{Nest}[f, x, 300]$. Then, to see whether the output is between 0.222 and 0.223 , define $h\left[x_{-}\right]:=\operatorname{If}[x \geq 0.222$ \&\& $x \leq 0.223,1$, $0]$. For a nice visual representation of which initial seeds lead to a payout on the 300 th pull, 


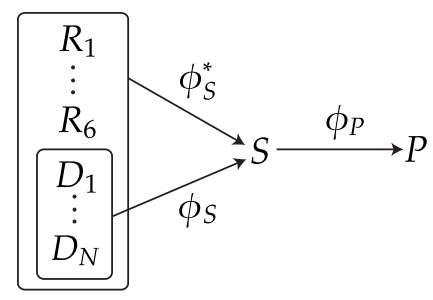

FIGURE 7: $\phi_{P}$ is a mapping from the potential values of the initial seed, $S$, to the value of $P . \phi_{S}$ is a mapping from the values of the initial conditions of the die roll, $D_{1}, \ldots, D_{N}$, to the value of $S . \phi_{S}^{*}$ is a mapping from the values of the randomly selected potential initial seeds, $R_{1}, \ldots, R_{6}$, and the initial conditions of the die roll, to the value of $S$.

Supposing that there is some chance that the slot machine pays out on the 3ooth pull, we could say any of the following four things about those chances:

I/6) there is a I/6 chance, and not a I/Iooo chance;

$\mathrm{I} / \mathrm{IOOO})$ there is a I/IOoo chance, and not a I/6 chance;

вотн) there is both a I/ 6 chance and a I/Iooo chance; or

NEITHER) there is neither a I/ 6 chance nor a I/IOoo chance.

If an account of deterministic chance endorses (вотн), say that it is a multivocal account. More generally, a multivocal account allows that a single token event may have multiple deterministic chances. If an account is not multivocal, then say that it is univocal. A univocal account of deterministic chance must endorse either (I/6), (I/IOOO), or (NEITHER).

Any multivocal account of chance incurs an explanatory burden. For, even if they are both created, the I/Ioooth chance and the I/6th chance are not created equal. Prima facie, if you know everything described in SLOT MACHINE, and no more, your credence that the machine pays out on the 300 th pull ought to be $\mathrm{I} / 6$, and it ought not be $\mathrm{I} / \mathrm{Io0}$. If you were told that there were 6,000 machines of which the description in SLOT MACHINE were true, you ought to expect about I,Ooo of them to pay out on the 3ooth pull; and you ought not expect about 6 of them to pay out on the 3 ooth pull. And a multivocal account of chance should do something to explain this asymmetry.

You might suspect that the obvious thing to say here is that we should side with the chances determined by earlier initial conditions over later ones for the purposes of prediction. For instance, you might think that we should have credence $\mathrm{I} / 6$, rather than $\mathrm{I} / \mathrm{IOOO}$, on the grounds that the initial conditions of the dynamics $\phi_{P} \circ \phi_{S}$ pre-date those of $\phi_{P} .{ }^{32}$ This approach won't work for sLOT

use DiscretePlot $[\mathrm{h}[\mathrm{g}[\mathrm{x}]],\{\mathrm{x}, 0,1,0.00001\}]$ to sample roo,ooo real numbers between $O$ and I, at steps of $0.0000 \mathrm{I}$, and see whether they lead to a payout on the 300 th pull. You'll see that about Ioo of them do, and those Ioo are randomly distributed over the interval between $O$ and I.

32 See Rosenthal (20I2, p. 23I), who suggests (but does not ultimately endorse-for reasons unre- 
MACHINE; for there is also the equation $\phi_{S}^{*}$ which is a function from the values of the six potential seeds $R_{1}, \ldots, R_{6}$ and the initial conditions of the die roll, $D_{1}, \ldots, D_{N}$, to the variable $S$. According to $\phi_{S}^{*}$, the value of $S$ is determined by both the values of the potential seeds and the initial conditions of the die roll. (We get $\phi_{S}$ from $\phi_{S}^{*}$ by simply plugging in the actual values of the variables $R_{1}, \ldots, R_{6}$.) See figure 7 . This dynamical equation has initial conditions which predate those of $\phi_{S}$, and the proportion of those initial conditions leading to a payout is approximately I/Iooo. Nevertheless, it does not appear that this deterministic chance trumps the deterministic chance of $\mathrm{I} / 6$ provided by the dynamics $\phi_{P} \circ \phi_{S}$. That is to say: given that we know all of this, it does not appear that we ought to have a credence of I/IOoO that the machine pays out on the 30oth pull. (For, to be clear, if we know all of this, then we know that $R_{3}$ is the only seed which leads to a payout on the 3ooth pull, and therefore, we know that the machine will pay out on the 300 th pull if and only if the die lands 3 up-and our credence in the latter ought to be $\mathrm{I} / 6$.)

There is a common prescription for making predictions when statistical data from multiple reference classes are available, due originally to ReICHENBACH (I97I). On this view, if you have statistics from multiple reference classes into which some token chance set up falls, then you ought to use the statistics from the narrowest reference class for the purposes of prediction. Applied to the current case, the proposal says that we should set our credence equal to the deterministic chance which would determine the narrowest reference class-i.e., the I/ 6 chance determined by $\phi_{P} \circ \phi_{S}$. Although I doubt that choosing the narrowest reference class is, in general, a good prescription, if we know that all of the frequencies from the various reference classes between which we have to choose align with some deterministic chance or other, then I endorse the advice to defer to the narrowest. However, providing a good general rule about how to defer to deterministic chances when more than one exists is not yet to do anything to explain why that general rule is a good one to follow. It would be preferable if we could explain why it is that we ought to defer to the chance which would determine the narrowest reference class. And one would hope that such an account would follow naturally from a story about the way in which deterministic chances constrain rational credence in general. Looking ahead, the account I will offer in $\S_{4}$ will provide us with a story like this.

lated to those in the body above) that, when we model cases like SLOT MACHINE with proximate dynamical equations like $\phi_{P}$, we have "overlooked a nomological factor relevant to the appearance of initial states and thus (indirectly) for the experimental outcomes. If we step further back and look how the initial states themselves come about, we should be able to discover this additional factor and re-model the experimental situation, this time explicitly paying attention to the neglected nomological influence... at some point, when we had taken all nomological factors relevant to the experimental result into account, we would finally arrive at a space in application to which [MAF yields] the correct outcome probabilities." 


\section{A Subjectivist Account of Deterministic Chance}

As I mentioned in the introduction, many accounts of deterministic chance built upon the MAF analysis endorse positions familiar from the history of philosophical theorizing about probability. For each of these accounts, deterministic chance is a certain kind of initial distribution filtered through appropriate causal dynamics. Some have followed the classical interpretation of probability in claiming that the initial distribution is, roughly, a uniform measure over the initial conditions space. ${ }^{33}$ Others have followed the actual/hypothetical frequency interpretation of probability in claiming that the initial distribution is an actual frequency distribution and/or the frequency distribution which very likely would result from a long series of trials. ${ }^{34}$ Others have attempted to utilize a 'best systems' account of probability to claim that the initial distribution is a law of nature. ${ }^{35}$

In the foregoing survey, one prominent player went missing: the personalist, or subjectivist. Traditional personalists like Ramsey (I93I), DE FinetTi (1974), and SAVAGE (I954) interpret probability claims as being about particular people's degrees of belief, or credences. SAVAGE (I97I) explicitly applies this understanding of probability to the method of arbitrary functions, effectively offering an account of deterministic chance according to which chance is just particular people's degrees of belief filtered through appropriate causal dynamics. His interpretation of the initial distributions as representing different individual's degrees of belief leads him to understand deterministic chance as a kind of wide intersubjective agreement among different people's credences. For instance, in case of the roulette wheel, Savage writes:

...people with very different opinions about $V$, as expressed by very different probability densities for $V$, may yet have nearly identical probability densities for black provided only that their opinions about $V$ are sufficiently diffuse... ${ }^{36}$

SAVAGE died before completing his article on deterministic chance, so it's unclear exactly how the account was to be fleshed out; but the account which he explicitly offers in the early pages is inadequate in at least three important respects. Firstly, SAVAGE does not solve the problem of all-too-arbitrary functions. For individuals may very well have spiky credence distributions like the one shown in figure 5-they may even do so reasonably, if they have evidence about the initial conditions of the dynamics in question. ${ }^{37}$ Secondly, as I noted in $\$ 2.3$, the PDP simply does not hold for an individual's credence function; nor does it hold for a rational individual's credence function. For those individuals may not have

33 Rosenthal (2010, 2012, 2016).

34 Strevens (2003, 20Ii), Abrams (20I2), and Beisbart (2016).

35 Albert (2000, 20I5), Loewer (200I, 2004, 2007), and Roberts (2016) (though Roberts is not committed to a 'best systems' account of laws).

36 SAVAGe (I97I, p. 420-2I, with slight notational changes)

37 "The conclusion does not apply at all to a person who feels quite sure of the second decimal of $V . ”($ SAVAGE, I97I, p. 42I, with notational changes) 
knowledge of the relevant dynamics, or they may have misleading evidence suggesting that the dynamics are different than they actually are (as, for instance, when an individual with knowledge of the dynamics of die rolls falsely believes a die to be fair). ${ }^{38}$ Thirdly, individuals need not, and in general do not, have opinions about the values of the variables which determine the value of the outcome variable $O$. Moreover, they need not, and in general do not, have any idea which variables determine the value of $O$.

Myrvold (20I2) provides a more sophisticated subjectivist account which advances on the first and third problems faced by SAVAGE's account. Firstly, he suggests that the initial distributions in MAF represent reasonable credences in the initial conditions of the chance outcome. He believes that this rules out all-tooarbitrary distributions like the one shown in figure 5 , for it would not be reasonable to have credences like those if one did not have any good evidence about the initial velocity of the roulette wheel's spin (which, he supposes, we in general cannot have). Secondly, he does not suppose that the credences in question are people's actual credences over the outcome variable-for those people may be ignorant of either the true dynamics or unopinionated about the initial values of the variables which determine $O$. Rather, the credence distributions in question are distributions that it would be reasonable to adopt, were the individuals informed about the relevant dynamics, and were they to become opinionated about the initial conditions and the outcome. Thus, we need not worry about the fact that most people do not actually have credences in the initial conditions of a coin flip, die roll, etc. Nor do we need to worry about the fact that, in the absence of information about the relevant dynamics, we should not expect even a reasonable credence function to satisfy the PDP-for the relevant initial distributions, on Myrvold's account, are all conditioned on the relevant dynamics.

These improvements help, but unfortunately not enough. Suppose that you have very good evidence that the roulette wheel is going to land on black-it doesn't matter for our purposes whether this evidence is misleading or not; misleading evidence is still evidence. Then, the distribution shown in figure 5 might be a reasonable credence distribution to adopt over the values of the variable $V$, were one to become informed of the dynamics and opinionated about the value of $V$. But even though evidence like this changes how confident you ought to be that the roulette wheel will stop on black, it does nothing to affect the chance that the roulette wheel stops on black.

I don't believe that this objection is fatal; and I wish to spend the remainder of this section developing a variant of the SAVAGE/Myrvold account which is capable of getting around this worry. The central innovation will be to replace MyrvolD's account-according to which chance is reasonable credence distributions filtered through the appropriate dynamics — with an account according to which chance is reasonable initial, or ur-prior, credence distributions, filtered through the appropriate dynamics. Restricting the account to reasonable ur-prior distributions

${ }_{38}$ See von Plato (I983, p. 42) 
allows us to get around the problem that a reasonable agent may have evidence about the initial conditions of $\phi_{O}$ and might, for this reason, adopt a spiky distribution like the one shown in figure 5. We rule such distributions out by only considering credence distributions which it would be permissible to adopt in the absence of any evidence.

\section{THE SUBJECTIVIST ACCOUNT}

For any correct, microconstant dynamical equation $\phi_{O}$ from $\left\langle C_{1}, \ldots, C_{N}\right\rangle$ to $O$, if $\llbracket \phi_{O} \rrbracket$ is the proposition that this dynamical equation is correct and $f$ is a reasonable initial (or 'ur-prior') credence distribution defined over $O, C_{1}, \ldots, C_{N}$, and $\llbracket \phi_{O} \rrbracket$, then,

$$
C h_{\phi_{O}}(O=o)=f\left(\phi^{-1}[O=o] \mid \llbracket \phi_{O} \rrbracket\right)
$$

In a slogan: chance is reasonable initial credence, conditioned on, and filtered through, dynamics. (As THE subjectivist ACCOUnT provides a multivocal account of chance-see $\$ 4.2$-I have explicitly indexed the chance function with the relevant dynamical equation, $\phi_{O}$. This will be relevant to my derivation of a deterministic principal principle and my treatment of SLOT MACHINE below.)

Let us assume that, in the absence of any evidence about the values of $C_{1}, \ldots$, $C_{N}$, spiky distributions like the one shown in figure 5 would be unreasonable. ${ }^{39}$ Then, THE SUBJECTIVE ACCOUNT solves the problem of all-too-arbitrary functions. Note that this solution does not depend upon anything nearly so strong as the principle of indifference - that, in the absence of evidence about $C_{1}, \ldots, C_{N}$, your credence distribution over $C_{1}, \ldots, C_{N}$ ought to be uniform. The subjective aCCOUNT is consistent with the thought that, in the absence of evidence, there is no credence distribution over $C_{1}, \ldots, C_{N}$ which is determinately rationally required. It does, however, require that certain very finely-discriminating credence distributions are determinately unreasonable in the absence of any relevant evidencee.g., ones for which the credence given to $\left[v, v^{*}\right]$ differs notably from the credence given to $\left[v+\epsilon, v^{*}+\epsilon\right]$, for small $\epsilon$.

Does the subjective account requires us to assume, with White (2005), that there is a unique credence distribution which is rationally required in the absense of evidence? As I will be understanding the account, it presupposes that there is some rational credence to adopt in any proposition in the absense of evidence — though it may be vague or indeterminate what that credence is. ${ }^{40}$ That

39 Why would they be unreasonable? I'm inclined to treat this as a datum for epistemology (pace radical subjectivist Bayesians), but we could justify it by appealing to a general normative principle like the following: your credences shouldn't strongly discriminate between very similar possibilities unless you have evidence which discriminates between these possibilities. (This is a strictly weaker principle than the principle of indifference (POI), and one that doesn't succumb to the usual objections to POI.)

40 Here, I am using 'indeterminate' in the sense that has become common in the literature on vagueness. You could be an epistemicist about this kind of indeterminacy, in which case you would understand me as saying: there is some one rational credence to adopt in any proposition 
is to say, there may not be a determinate fact of the matter which credence distribution over initial conditions we ought to have. For instance, in the MAF analysis of the deterministic chance of a coin flip landing heads, it could be indeterminate whether $f$ gives probability 0.5000 I or 0.49999 to the values of $\langle U, A\rangle$ which lead to the coin landing heads. Nevertheless, it could still be determinate that $f$ does not give probability 0.6 to those values. ${ }^{4 \mathrm{I}}$ Assuming a standard semantics for determinacy, then, THE sUBJECTIVIST ACCOUNT is committed to something like WHITE (2005)'s uniqueness thesis, according to which there is one and only one doxastic state which is rational given any body of evidence. However, while this view agrees with WHITE that it is determinate that there is one rational credence to adopt given no evidence, it denies that there is any credence which it is determinately rational to adopt given no evidence. ${ }^{42}$

\section{I A Deterministic Principal Principle}

In the presence of some rather weak normative assumptions, THE SUBJECTIVIST ACCOUnT entails a deterministic variant of LeWIs (I980)'s Principal Principle. In this section, I will lay out these normative assumptions and show how they can be used to derive a deterministic principal principle from THE SUBJECTIVIST ACCount. As we go, it will be important to clearly distinguish reasonable initial credence distributions which are defined over $C_{1}, \ldots, C_{N}$ and $\llbracket \phi_{O} \rrbracket$ from those which are not. Throughout this section, then, I will use ' $f$ ' to stand for a reasonable initial credence function defined over $O$. A reasonable initial credence function defined over $O$ and $\llbracket \phi_{O} \rrbracket$ I will denote with ' $f_{\phi}$ ' $\cdot f_{\phi}$, then, is opinionated about the outcome variable as well as the dynamics determining the value

in the absense of evidence-though nobody can know what it is. Or you may think that this kind of indeterminacy is due to an unsettledness in the way we use language, or that there's something genuinely unsettled about normative reality. See Williamson (I994) for more on different theories of indeterminacy.

4I There is a common framework for representing indeterminate probabilities like these (see vAN FraAssen (I990, 2006), LeVi (I974), Walley (I99i), Joyce (20I0), and White (2009)). In this framework, we would take all the admissible candidates for $f$ and gather them into a set, call it ' $\mathcal{F}$ '. We would then use $\mathcal{F}$ to represent a reasonable initial doxastic state. The probabilities included in $\mathcal{F}$ are akin to the admissible precisifications in supervaluationist theories of vagueness (see FIne (1975) and Keefe (2000)). While a supervaluationist keeps these admissible precisifications in their metalinguistic interpretation of a theory, the imprecise probabilist uses them in their first-order theorizing. From my perspective, it is better to handle indeterminacy with respect to reasonable credence in the same way that other indeterminacy is handled, and to keep the admissible precisifications in the meta-language.

42 To appreciate the distinction in scope here, consider the sorites argument. If you accept that classical logic is determinately true, then you'll accept that it is determinately the case that there is an $n$ such that $n$ is the least number of grains that makes a heap. However, if you think that it's indeterminate when some grains go from a heap to a non-heap, then you'll deny that there is any number $n$ which is determinately the least number of grains which makes a heap. Similarly, I am suggesting that it's determinately the case that there is one and only one rational credence to adopt in the absence of evidence; though it's indeterminate which credence it is, so there is no credence which is determinately the one and only rational credence to adopt in the absence of evidence. 
of $O$-though perhaps not the value of the initial conditions $C_{1}, \ldots, C_{N}$ which determine the value of $O$, according to $\phi_{O}$. Finally, a reasonable initial credence function defined over $O, \llbracket \phi_{O} \rrbracket$, and the initial conditions $C_{1}, \ldots, C_{N}$, I will denote with ' $f_{\phi, C}$ '.

It will also be important, as we go, to clearly distinguish the claim that the $\phi_{O^{-}}$ chance of $O=o$ is $x$ - a proposition we can denote with ' $\llbracket C h_{\phi_{O}}(O=o)=x \rrbracket$ 'from the claim that there is an $x$ chance of $O=o-$ a proposition we can denote

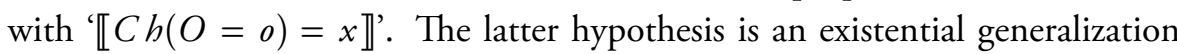
over hypotheses like the former.

$$
\llbracket C h(O=o)=x \rrbracket \Longleftrightarrow \exists \phi_{O}: \llbracket C h_{\phi_{O}}(O=o)=x \rrbracket
$$

Keeping these propositions separate will be important, since I will first derive what I will call the particular deterministic principal principle, which says that, for any particular equation $\phi_{O}$, your credence in $O=o$, given $\llbracket C h_{\phi_{O}}(O=o)=x \rrbracket$, and given any admissible evidence, ought to be $x$. I will then derive what I will call the general deterministic principal principle, which says that your credence in $O=o$, given that there is an $x$ chance of $O=o, \llbracket C h(O=o)=x \rrbracket$, and given any admissible evidence, ought to be $x$.

According to THE SUBJECTIVIST ACCOUNT, if we have a microconstant dynamical equation $\phi_{O}$, then the claim that the $\phi_{O}$-chance of $O=o$ is $x$ is true if and only if both $\phi_{O}$ is correct and $f_{\phi, C}$ assigns a credence of $x$ to the proposition $\phi_{O}^{-1}[O=o]$, once $f_{\phi, C}$ is conditioned on the true proposition $\llbracket \phi_{O} \rrbracket$. Let's denote the proposition that $f_{\phi, C}$ assigns a credence of $x$ to $A$, given that $E$, with ' $\llbracket f_{\phi, C}(A \mid E)=x \rrbracket$ '. Then, the subjectivist account tells us that, for some microconstant $\phi_{O}, 43$

(I) $\llbracket C h_{\phi_{O}}(O=o)=x \rrbracket \Longleftrightarrow \llbracket f_{\phi, C}\left(\phi_{O}^{-1}[O=o] \mid \llbracket \phi_{O} \rrbracket\right)=x \rrbracket \wedge \llbracket \phi_{O} \rrbracket$

Given some weak normative assumptions, we can now derive a deterministic variant of the principal principle. The first normative principle we will require is what we can call a 'causal induction' principle. This principle says that, conditional on only the dynamical equation, $\llbracket \phi_{O} \rrbracket, f_{\phi, C}$ would satisfy the PDP,

\section{CAUSAL INDUCTION}

$$
f_{\phi, C}\left(\phi_{O}^{-1}[O=o] \mid \llbracket \phi_{O} \rrbracket\right)=f_{\phi, C}\left(O=o \mid \llbracket \phi_{O} \rrbracket\right)
$$

Causal induction is an incredibly weak principle. It follows from probabilism alone. For the proposition $\llbracket \phi_{O} \rrbracket$ entails that $\phi_{O}^{-1}[O=o] \Leftrightarrow O=o$. And it follows from probabilism alone that, conditional on $A \Leftrightarrow B$, your credence in $A$ should be equal to your credence in $B$. So causal induction should not be controversial.

43 A comment on notation: I will denote the conjunction of propositions $p$ and $q$ with both ' $p \wedge q$ ' and ' $p q$ '. 
Causal induction and (I) together entail (2):

$$
\llbracket C h_{\phi_{O}}(O=o)=x \rrbracket \Longleftrightarrow \llbracket f_{\phi, C}\left(O=o \mid \llbracket \phi_{O} \rrbracket\right)=x \rrbracket \wedge \llbracket \phi_{O} \rrbracket
$$

Looking at (2), we can see that, according to THE SUBJECTIVIST ACCOUNT, the claim that the deterministic $\phi_{O}$-chance of $O=o$ is $x$ is the conjunction of two claims: the first is a normative claim about which credence distribution is rational in the absence of any evidence; and the second, a descriptive claim about the world's causal dynamics. Uncertainty about the chances could therefore result from uncertainty about either of these conjuncts.

The second normative principle we will require is an enkratic principle which says how uncertainty about the normative component of a deterministic chance constrains reasonable credence. The principle says that, where $E$ is any evidence consistent with it being the case that an initial credence that $A$, given $E$, ought to be $x$, a reasonable initial credence that $A$, given $E$ and given that an initial credence in $A$, given $E$, ought to be $x$, is $x .{ }^{44}$

\section{ENKRATIC PRINCIPLE}

Where ' $f$ ' ' is a rational initial credence function defined over $A$ and $E$, and $f$ is a rational initial credence defined over $A, E$, and $\llbracket f^{*}(A \mid$ $E)=x \rrbracket$,

$$
f\left(A \mid \llbracket f^{*}(A \mid E)=x \rrbracket \wedge E\right)=x, \text { if defined }
$$

There are reasons to worry about enkratic principles in general. For instance, if a certain kind of externalism about evidence is correct, then information about which response to your evidence is rational can provide additional information about what your evidence is. ${ }^{45}$ Then, even though $x$ may be the rational credence to have in $A$ given $E$, the additional information provided by the normative proposition $\llbracket f^{*}(A \mid E)=x \rrbracket$ may make it rational to have a credence other than $x$ in $A$. In response to cases like those, ElGA (2013) has argued that principles like ENKRATIC PRINCIPLE need to be generalized. However, those problem cases - if they are problem cases_-only cause problems for enkratic principles that say something about how knowledge that $f$ is the rational credence function to adopt in your very epistemic circumstances ought to impact your current credences. ENKRATIC PRINCIPLE says nothing about that. Rather, it only says something about what a reasonable ur-prior credence is, given only information about what a reasonable ur-prior credence is. And the information that a reasonable ur-prior is thus-and-so couldn't possibly convey anything about what evidence has been acquired, since reasonable ur-priors are the priors that it would be reasonable to

44 When principles like ENKRATIC PRINCIPLE show up in the literature-e.g., in ElGA (20I3) - they are often formulated with a proposition like $\llbracket f=\mathrm{f} \rrbracket$, which says that $\mathrm{f}$ is a reasonable initial credence function. Then, authors state the enkratic requirement as follows: $f(A \mid \llbracket f=\mathrm{f} \rrbracket)=$ $\mathrm{f}(A)$. The ENKRATIC PRINCIPLE in the body is strictly weaker than this principle.

45 See Williamson (2000, 20II, 2014). 
adopt in the absence of any evidence.

We may worry about instances of ENKRATIC PRINCIPLE where $\llbracket f^{*}(A \mid E)=$ $x \rrbracket$ is false. Perhaps, conditional on the false proposition that a certain initial credence that $A$, given $E$, is rational, a rational initial credence function should stick to its guns and have whatever credence in $A$, conditional on $E$, really is rational. Those who hold that uncertainty about the a priori requirements of rationality is itself irrational ${ }^{46}$ will be attracted to this position. For such philosophers, however, I need not appeal to ENKRATIC PRINCIPLE in the first place. According to THE SUbJECTIVIST ACCOUNT, facts about deterministic chances are partly descriptivethey are in part to do with the worldly dynamics—and partly normative- they are in part to do with the credal distributions it would be rational to hold in the absence of evidence. Uncertainty about the chances is therefore uncertainty about either the worldly dynamics or the a priori requirements of rationality, or both. If you hold that uncertainty about the a priori requirements of rationality is rationally impermissible, then you will think that, for all true normative propositions of the form $\llbracket f^{*}(A \mid E)=x \rrbracket$, a rational credence function $f$ will be certain of these propositions, and all uncertainty about deterministic chance will be uncertainty about the worldly dynamics. This will suffice for my purposes here.

There is another position which denies ENKRATIC PRINCIPLE, however, which will not suffice for my purposes here. On that view, uncertainty about the a priori requirements of rationality is rationally permissible, but indicative suppositions about the requirements of rationality does nothing to affect those requirements. So, even though it may be permissible for you have a non-zero credence in the false proposition that any initial credence that $A$ between I/ 4 and I/ 3 is permissible, so long as no such credence actually is permissible, you are irrational if your credence that $A$ is between $\mathrm{I} / 4$ and $\mathrm{I} / 3$, conditional on that false proposition. I find such 'level-splitting' views somewhat plausible in cases in which you face uncertainty about your current evidence-but I do not find them plausible when it comes to the rational requirements placed on an initial, ur-prior credence function. ${ }^{47}$ If you are beholden to a view like this, then I am afraid that I must leave you behind at this point.

Note that (3) is an instance of ENKRATIC PRINCIPLE, where we have replaced ' $A$ ' with ' $O=o^{\prime}$, ' $E$ ' with '【 $\phi_{O} \rrbracket$ ', ' $f$ ' with ' $f_{\phi}$ ', and ' $f^{*}$ ' with ' $f_{\phi, C}$ '.

$$
f_{\phi}\left(O=o \mid \llbracket f_{\phi, C}\left(O=o \mid \llbracket \phi_{O} \rrbracket\right)=x \rrbracket \wedge \llbracket \phi_{O} \rrbracket\right)=x
$$

${ }^{46}$ See Titelbaum (20I4), who argues for this view from a principle slightly stronger than what I have named ENKRATIC PRINCIPLE in the body. Throughout, by 'a priori requirements of rationality', I just mean the constraints which rationality places on our doxastic states in the absence of evidence.

47 For more discussion, see Lasonen-Aarnio (20I4, 20I5, forthcoming), Horowitz (20I4), Greco (20I4), and Titelbaum (20I4). 
And (2) and (3) imply (4).

$$
f_{\phi}\left(O=o \mid \llbracket C h_{\phi_{O}}(O=o)=x \rrbracket\right)=x
$$

We will say that evidence $E$ is $\phi_{O}$-admissible iff, given knowledge of the normative proposition $\llbracket f_{\phi, C}\left(O=o \mid \llbracket \phi_{O} \rrbracket\right)=x \rrbracket$ and the dynamical equation $\llbracket \phi_{O} \rrbracket, E$ is probabilistically independent of $O=o$ according to $f_{\phi}$.

\section{Admissibility}

Total evidence $E$ is $\phi_{O}$-admissible just in case

$$
\begin{aligned}
& f_{\phi}\left(O=o \mid \llbracket f_{\phi, C}\left(O=o \mid \llbracket \phi_{O} \rrbracket\right)=x \rrbracket \wedge \llbracket \phi_{O} \rrbracket \wedge E\right) \\
= & f_{\phi}\left(O=o \mid \llbracket f_{\phi, C}\left(O=o \mid \llbracket \phi_{O} \rrbracket\right)=x \rrbracket \wedge \llbracket \phi_{O} \rrbracket\right)
\end{aligned}
$$

Admissibility is relative to a dynamical equation, $\phi_{O}$. Bodies of evidence $E$ are $\phi_{O}$-admissible provided that, given $\llbracket \phi_{O} \rrbracket$ and $\llbracket f\left(O=o \mid \llbracket \phi_{O} \rrbracket\right)=x \rrbracket$, they do not provide information about the outcome $O=o$. So, for instance, evidence supporting the hypothesis that this particular roulette wheel spin lands on black is inadmissible relative to $\phi_{B}$. So too is any evidence supporting the hypothesis that the initial velocity of the roulette wheel spin is between 4.15 and $4.16 \mathrm{~m} / \mathrm{s}$, since, given $\llbracket \phi_{B} \rrbracket$, such evidence would lead $f_{\phi, C}$ to rationally change its credence that the roulette wheel stops on black. It doesn't matter, in the definition of admissibility, whether this evidence is misleading or not. All that matters is whether a reasonable initial credence function would change its credence in $O=o$ in response to the evidence. ${ }^{48}$

Given this definition of admissibility, (2) and (3) imply that, for any admissible evidence $E$,

$$
f_{\phi}\left(O=o \mid \llbracket C h_{\phi_{O}}(O=o)=x \rrbracket \wedge E\right)=x
$$

(5) gives us a relation between deterministic chance propositions and rational credence. I will call this constraint the particular deterministic principal principle (to be contrasted with the general deterministic principal principle below). Putting the principle in a form familiar from LewIs (1980), it says:

\section{PARTICULAR DETERMINISTIC PRINCIPAL PRINCIPLE}

If $A$ is the proposition that an outcome variable $O$ takes on the value $o, X$ is the proposition that the $\phi_{O^{-}}$-hance of $A$ is $x, E$ is any $\phi_{O^{-}}$ admissible evidence, and $f_{\phi}$ is a reasonable initial credence function defined over $A, X$, and $E$, then

$$
f_{\phi}(A \mid X E)=x
$$

$4^{8}$ Admissibility is defined relative to bodies of total evidence; for, given this definition of admissibility, admissibility need not agglomerate — simply because $E_{1}$ is admissible and $E_{2}$ is admissible, this needn't mean that $E_{1} \wedge E_{2}$ is admissible. 
This version of the principal principle differs from LewIs's original formulation of his tychistic principal principle in two respects: firstly, LEwIs's original formulation of the principal principle used time to provide a sufficient condition 49 for the admissibility of evidence relative to a chance ( $v i z$, the past at $t$ is admissible relative to the time $t$ chances), whereas the variant above gives a necessary and sufficient condition on the admissibility of evidence relative to a chance in terms of that evidence's effect on reasonable initial credence functions. Secondly, PARTICULAR DETERMINISTIC PRINCIPAL PRINCIPLE concerns the chance hypothesis that a particular chance of $A$ is $x$, rather than the general hypothesis that there is an $x$ chance of $A$. Because THE SUBjectivist AcCount is a multivocal account of chance (see $\$ 4.2$ ), there may be cases in which there are multiple deterministic chances that $A$. Particular Deterministic Principal Principle tells us that, conditional on any of one them and any evidence which is admissible relative to that chance, our credence that $A$ should align with that chance.

PARTICULAR DETERMINISTIC PRINCIPAL PRINCIPLE is all the principal principle I will require in order to handle cases like sLot MACHIne (\$4.2) and to show that particular deterministic chances can explain frequency data $(\$ 4.4)$. But it is reasonable to want more than it provides. In both everyday life and in statistical applications, we entertain deterministic chance hypotheses without having any idea what dynamical equation might underlie these chance hypotheses. So it would be nice if we could additionally vindicate what I will call the general deterministic principal principle.

\section{GENERAL DETERMINISTIC PRINCIPAL PRINCIPLE}

If $A$ is the proposition that an outcome variable $O$ takes on the value $o, X$ is the proposition that there is a deterministic $x$ chance of $A, E$ is any $A X$-admissible evidence, and $f$ is a reasonable initial credence function defined over $A, X$, and $E$, then

$$
f(A \mid X E)=x
$$

The particular deterministic principal principle enjoins you to have credence $x$ in $A$, given that a particular dynamical equation $\phi$ determines an $x$ chance for $A$. The general principal principle tells you to do the same when you know only that some dynamical equation determines an $x$ chance for $A$.

Note that, in the statement of GENERAL DETERMINISTIC PRINCIPAL PRINCIPLE, admissibility is defined relative to the proposition $A$ and the chance hypothesis $X$. We will say that the total evidence $E$ is admissible, relative to a chance outcome $O=o$ and a general chance hypothesis $\llbracket C h(O=o)=x \rrbracket$, iff $E$ is admissible relative to every particular chance hypothesis $\llbracket C h_{\phi_{O}}(O=o)=x \rrbracket$, and it is also admissible relative to every conjunction of such hypotheses.

49 Or, rather, an "almost sufficient" condition—a qualification Lewis included due to worries about news from the future. 


\section{GENERAL ADMISSIBILITY}

Total evidence $E$ is $O=o, x$-admissible just in case, for any conjunction of particular chance hypotheses which generates an $x$ chance for $O=o, X_{1} \ldots X_{N}, E$ is probabilistically independent of $O=o$, given $X_{1} \ldots X_{N}$,

$$
f_{\phi}\left(O=o \mid X_{1} \ldots X_{N} E\right)=f_{\phi}\left(O=o \mid X_{1} \ldots X_{N}\right)
$$

Given this understanding of admissibility, at least in the special case where there are at most finitely many potential particular deterministic chances, we may derive GENERAL DETERMINISTIC PRINCIPAL PRINCIPLE from the PARTICULAR DETERMINISTIC PRINCIPAL PRINCIPLE with one additional assumption. In what follows, let ' $A$ ' be the proposition that $O=o$, and let ' $X_{1}$ ', ' $X_{2}$ ', and so on be claims of the form $\llbracket C h_{\phi}(A)=x \rrbracket$ (with fixed $A$ and $x$ and variable $\phi$ ). Then, the one additional assumption I will need to make is that, for any $X_{1}, X_{2}, \ldots, X_{N}$, given that each of $X_{1}$ through $X_{N}$ is true, your credence in $A$ is $x$.

$$
f_{\phi}\left(A \mid X_{1} X_{2} \cdots X_{N}\right)=x
$$

Then, we may verify that, for any $N$ chance hypotheses $X_{1}, \ldots, X_{N}$, and any generally admissible evidence $E$,

$$
f_{\phi}\left(A \mid\left(X_{1} \vee \cdots \vee X_{N}\right) E\right)=x
$$

To see that this is so, begin by asking whether

$$
\begin{gathered}
f_{\phi}\left(A \mid E X_{1} \vee \cdots \vee E X_{N}\right) \stackrel{?}{=} x \\
\frac{f_{\phi}\left(A E X_{1} \vee \cdots \vee A E X_{N}\right)}{f_{\phi}\left(E X_{1} \vee \cdots \vee E X_{N}\right)} \stackrel{?}{=} x \\
f_{\phi}\left(A E X_{1} \vee \cdots \vee A E X_{N}\right) \stackrel{?}{=} x \cdot f_{\phi}\left(E X_{1} \vee \cdots \vee E X_{N}\right) \\
\text { (7) } \sum_{k=1}^{N}(-1)^{k-1} \sum_{i \in \mathfrak{X}_{k}} f_{\phi}\left(A E X_{i_{1}} \cdots X_{i_{k}}\right) \stackrel{?}{=} x \cdot \sum_{k=1}^{N}(-1)^{k-1} \sum_{i \in \mathfrak{X}_{k}} f_{\phi}\left(E X_{i_{1}} \cdots X_{i_{k}}\right)
\end{gathered}
$$

Where ' $\mathfrak{X}_{k}$ ' is the set of all $k$-membered subsets of $\left\{X_{1}, X_{2}, \ldots, X_{N}\right\}$, and ' $X_{i_{j}}$ ' is the $j$ th member of $i \in \mathfrak{X}_{k}$ (given some enumeration). The definition of general admissibility tells us that $E$ is independent of $A$ conditional on any conjunction of chance hypotheses $X_{i_{1}} \cdots X_{i_{k}}$, so the left-hand-side of (7) is

$$
\sum_{k=1}^{N}(-1)^{k-1} \sum_{i \in \mathfrak{X}_{k}} f_{\phi}\left(A \mid X_{i_{1}} \cdots X_{i_{k}}\right) \cdot f_{\phi}\left(E X_{i_{1}} \cdots X_{i_{k}}\right)
$$


By (6), we have that $f_{\phi}\left(A \mid X_{i_{1}} \cdots X_{i_{k}}\right)=x$, so (7) reduces to the trivial equality

$$
x \cdot \sum_{k=1}^{N}(-1)^{k-1} \sum_{i \in \mathfrak{X}_{k}} f_{\phi}\left(E X_{i_{1}} \cdots X_{i_{k}}\right) \stackrel{\checkmark}{=} x \cdot \sum_{k=1}^{N}(-1)^{k-1} \sum_{i \in \mathfrak{X}_{k}} f_{\phi}\left(E X_{i_{1}} \cdots X_{i_{k}}\right)
$$

So, at least in the special case where there are at most finitely many potential particular deterministic chances, and granting the assumption (6), the general chance hypothesis $\llbracket C h(A)=x \rrbracket$ entails that $\llbracket f_{\phi}(A \mid \llbracket C h(A)=x \rrbracket \wedge E)=x \rrbracket$ when $E$ is admissible. Therefore, the conjunction

$$
\llbracket C h(A)=x \rrbracket \wedge \llbracket f_{\phi}(A \mid \llbracket C h(A)=x \rrbracket \wedge E)=x \rrbracket
$$

is equivalent to $\llbracket C h(A)=x \rrbracket$. So a reasonable credence in $A$ given that the chance of $A$ is $x$ and $E$ must be equal to a reasonable credence that $A$ given the conjunction (8) and $E$.

$$
\begin{aligned}
& f(A \mid \llbracket C h(A)=x \rrbracket \wedge E) \\
= & f\left(A \mid \llbracket C h(A)=x \rrbracket \wedge \llbracket f_{\phi}(A \mid \llbracket C h(A)=x \rrbracket \wedge E)=x \rrbracket \wedge E\right)
\end{aligned}
$$

The ENKRATIC PRINCIPLE then tells us that

$$
f\left(A \mid \llbracket f_{\phi}(A \mid \llbracket C h(A)=x \rrbracket \wedge E)=x \rrbracket \wedge \llbracket C h(A)=x \rrbracket \wedge E\right)=x
$$

And, from (9) and (Io), we have that

$$
f(A \mid \llbracket C h(A)=x \rrbracket \wedge E)=x
$$

which is the GENERAL DETERMINISTIC PRINCIPAL PRINCIPLE.

The subjectivist aCCOUnT thus abides by Lewis's maxim:

Don't call any alleged feature of reality "chance" unless you've already shown that you have something, knowledge of which could constrain rational credence. ${ }^{50}$

Note that once we have the deterministic principal principle, we have all that we need in order to explain how knowledge of frequencies could provide information about deterministic chance. For this principle tells us that, if our initial credences are reasonable, and if we update those credences on frequency data by conditionalization, then chance hypotheses which make that data more likely will be confirmed to a greater degree, and chance hypotheses which make that data less likely will be confirmed to a lesser degree. ${ }^{\text {I }}$ On the subjectivist account, information about the frequency of heads landings can end up providing us with information about how confident a better informed, rational inquirer would be that the next coin toss will land heads. We can come by this information without

so LewIS (I994, p. 484)

sI See Lewis (I980, pp. 285-287). 


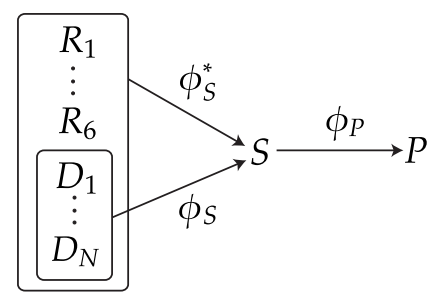

Figure 7: $\phi_{P}$ is a mapping from the potential values of the initial seed, $S$, to the value of $P . \phi_{S}$ is a mapping from the values of the initial conditions of the die roll, $D_{1}, \ldots, D_{N}$, to the value of $S . \phi_{S}^{*}$ is a mapping from the values of the potential initial seeds, $R_{1}, \ldots, R_{6}$, and the initial conditions of the die roll, to the value of $S$.

thereby coming to know anything about the underlying dynamics which inform this merely hypothetical agent's credences. ${ }^{52,53}$

\subsection{Slot Machine}

As I will understand it, THE SUBJECTIVIST ACCOUNT is an account of token chance. I also assume that a single token outcome may be correctly described by multiple correct dynamical equations. Then, THE subjectivist ACCOUnt is a multivocal account of chance. In SLOT MACHINE, there is a true dynamical equation, $\phi_{P}$, according to which the outcome variable $P$ is determined by the value of the initial seed, $S$. There is another true dynamical equation, $\phi_{P} \circ \phi_{S}$, according to which the outcome variable $P$ is determined by the initial conditions of the die roll, $D_{1}, \ldots, D_{N}$. And there is a third true dynamical equation, $\phi_{P} \circ \phi_{S}^{*}$, according to which the outcome variable $P$ is determined by both the initial conditions of the die roll and the potential seeds, $R_{1}, \ldots, R_{6}$.

(See figure 7, reproduced above.) If we suppose that a reasonable initial credence distribution defined over $S$, and conditionalized only on the proposition $\llbracket \phi_{P} \rrbracket$, would place about I/Ioooth of its mass on values of $S$ leading to a payout on the 3ooth pull, then there will be a I/Iooo chance of the machine paying out, determined by the equation $\phi_{P}$. If we additionally suppose that a reasonable initial credence distributions defined over the initial conditions of the die roll, $D_{1}, \ldots, D_{N}$, and conditionalized only on the proposition $\llbracket \phi_{P} \circ \phi_{S} \rrbracket$, would place about $\mathrm{I} / 6$ th of its mass on values leading to a payout on the 300 th pull, then there will be a I/ 6 chance of the machine paying out, determined by the equation $\phi_{P} \circ \phi_{S}$. Finally, if we suppose that a reasonable initial credence distributions defined over both the potential initial seed values and the initial conditions of the die roll, and conditionalized only on the proposition $\llbracket \phi_{P} \circ \phi_{S}^{*} \rrbracket$, would place

52 Of course, in order to come to know that the deterministic chance of a coin landing heads is $\mathrm{I} / 2$, we must antecedently know that there is a deterministic chance that the coin lands heads.

53 An interesting case to consider arises when we are not uncertain about the dynamics, but we are uncertain about the requirements of rationality. If we take such cases to be possible then we could acquire a posteriori confirmation of normative propositions about the requirements of rationality by observing frequency data. 
about I/roooth of its mass on values leading to a payout on the 300 th pull, then there will be (another) I/IOOO chance of the machine paying out, determined by the equation $\phi_{P} \circ \phi_{S}^{*}$. The SUBJECTIVIST ACCOUnT thus endorses answer (вотн) in SLOT MACHINE.

The SUbJECTIVIST ACCOUNT additionally provides a clear explanation of why one of these chances should hold pride of place for the purposes of prediction, given that you know everything described in SLOT MACHINE - that is, it explains why, given that you know everything described in SLOT MACHINE, you should have credence $\mathrm{I} / 6$ that the machine pays out. Recall that, in SLOT MACHINE, you know that exactly one of the pre-selected seeds will lead to a payout on the 300 th pull. If you know this, then you know $\llbracket \phi_{P} \circ \phi_{S} \rrbracket$. And $\llbracket \phi_{P} \circ \phi_{S} \rrbracket$ is inadmissible information for both for the I/Iooo chance determined by $\phi_{P}$ and for the I/Iooo chance determined by $\phi_{P} \circ \phi_{S}^{*}$. A reasonable initial credence function, conditioned on the setup of the case, will regard the information that the initial seed value was determined by a die roll in such a way that, if the die lands 3 up, the initial seed value gets mapped to a payout on the 300 th pull (the information provided by $\left.\llbracket \phi_{P} \circ \phi_{S} \rrbracket\right)$ as being probabilistically relevant to the value of the initial seed, $S$. In the presence of $\llbracket \phi_{P} \rrbracket$, $\llbracket \phi_{P} \circ \phi_{S} \rrbracket$ provides information about the value of $S$, and thus, provides information about the initial conditions of the dynamical equation $\phi_{P}$. The proposition $\llbracket \phi_{P} \circ \phi_{S} \rrbracket$ is therefore inadmissible relative to the chance determined by $\phi_{P}$. Similarly, in the presence of the proposition $\llbracket \phi_{P} \circ \phi_{S}^{*} \rrbracket$, the information that iff the die lands 3 up, the initial seed value will be mapped to a payout on the 3ooth pull, entails that $R_{3}$ is the only potential seed which gets mapped to a payout. Thus, in the presence of $\llbracket \phi_{P} \circ \phi_{S}^{*} \rrbracket$, $\llbracket \phi_{P} \circ \phi_{S} \rrbracket$ provides information about the initial conditions of $\phi_{P} \circ \phi_{S}^{*}$, and is therefore inadmissible relative to the chance determined by $\phi_{P} \circ \phi_{S}^{*}$.

The same thing cannot be said in the other direction. $\llbracket \phi_{P} \rrbracket$ does not provide information which is inadmissible for the chance determined by $\phi_{P} \circ \phi_{S}$. Nor does $\llbracket \phi_{P} \circ \phi_{S}^{*} \rrbracket$ provide information which is inadmissible for the chance determined by $\phi_{P} \circ \phi_{S}$. So, while your information is inadmissible relative to both of the I/roooth chances, it is admissible relative to the I/6th chance. So the PARTICULAR DETERMINISTIC PRINCIPAL PRINCIPLE will not tell us to defer to the I/Ioooth chances; whereas, it will tell us to defer to the I/6th chance. And, given our normative assumptions, THE SUBJECTIVIST ACCOUNT entails the PARTICULAR DETERMINISTIC PRINCIPAL PRINCIPLE. So-and this is the point-THE SUBJECTIVIST ACCOUNT is able to explain why you should have a credence of $\mathrm{I} / 6 \mathrm{th}$, and not I/roooth, that the machine pays out. It explains why, though you know about all three of these deterministic chances, only one of them should guide your predictions. ${ }^{54}$

54 This only holds if you know that exactly one of the pre-selected seeds leads to a payout on the 3ooth pull. Without this knowledge, you will not know about the $\mathrm{I} / 6$ th chance determined by $\llbracket \phi_{P} \circ \phi_{S} \rrbracket$. In that case, the PARTICULAR DETERMINISTIC PRINCIPAL PRINCIPLE will tell you that you should have a credence of I/IOoO that the machine pays out. 

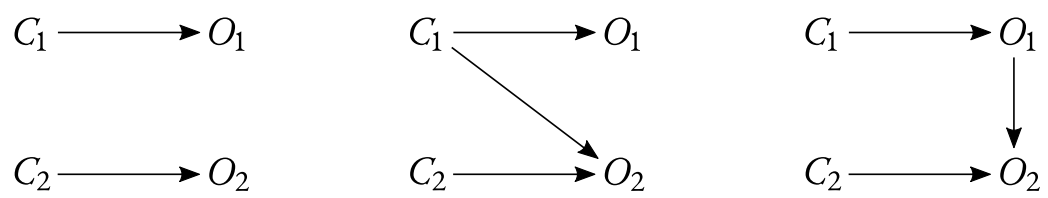

FIGURE 8: In the diagrams above, an arrow is drawn from one variable to another just in case the value of the variable at the tail of the arrow causally determines the value of the variable at the tip. In the far left diagram, $O_{1}$ and $O_{2}$ are causally independent; in the other two diagrams, they are not.

\subsection{INDEPENDENCE}

So far, I have just been talking about the chance of single outcomes. What is it, according to THE SUBJECTIVIST ACCOUNT, for the chance of two token outcomes to be independent or dependent? According to the subjectivist account, the independence of two token outcomes $O_{1}$ and $O_{2}$ is just a property of the chance distribution over $\left\langle O_{1}, O_{2}\right\rangle$; and this chance distribution is determined in the same way as every other chance distribution: find some true dynamical equations which determine the values of $O_{1}$ and $O_{2}$ and look at the reasonable credence assignments to the independent variables in those equations. In a case where we are considering two outcome variables, however, we must move from talking about a single dynamical equation, $O:=\phi_{O}\left(C_{1}, \ldots, C_{N}\right)$, to talking about a system of dynamical equations,

$$
\begin{aligned}
O_{1} & :=\phi_{O_{1}}\left(C_{1}^{1}, \ldots, C_{N}^{1}\right) \\
O_{2} & :=\phi_{O_{2}}\left(C_{1}^{2}, \ldots, C_{M}^{2}\right)
\end{aligned}
$$

If we have a system of equations according to which $O_{1}$ and $O_{2}$ are causally independent, then a macroperiodic credence distribution over the causes of $O_{1}$ and $\mathrm{O}_{2}$ will induce, via the (PDP), a joint distribution over $O_{1}$ and $O_{2}$ on which those variables are probabilistically independent of one another. If, on the other hand, we have a system of equations according to which $O_{1}$ and $O_{2}$ are not causally independent, then a macroperiodic credence distribution over the causes of $O_{1}$ and $O_{2}$ may induce, via the (PDP), a joint distribution on which $O_{1}$ and $O_{2}$ are probabilistically dependent.

\subsection{Explanation}

When it comes to the matter of explaining frequency data by appeal to chances, there are two philosophical camps. In one camp are those who think that high and low chances alike may explain. In the other camp are those who think that, while high chances may explain, low chances may not. Following STREVENs (2000), call those in the first camp 'egalitarians', and call those in the second camp 'elitists'. To illustrate: suppose that we toss a fair $€ \mathrm{I}$ coin and a fair $£ \mathrm{I}$ coin I,O0O times each. The $€ \mathrm{I}$ coin lands heads around 500 times (plus or minus 30 ), and the $£ \mathrm{I}$ 
coin never lands heads. Consider the two explanations:

E) The $£$ I coin never landed heads because there was a $(\mathrm{I} / 2)^{\mathrm{I}, \mathrm{ooo}}$ chance that that would happen.

$€)$ The $€$ I coin landed heads about half the time because there was around a $95 \%$ chance that that would happen.

Elitists think that only $(€)$ is a good explanation, whereas egalitarians think that both $(€)$ and $(£)$ are good explanations. Moderate egalitarians may say that, while $(€)$ and $(£)$ are both good explanations, $(€)$ is a much better explanation than $(£)$.

One motivation for elitism and moderate egalitarianism comes from a nomic expectability requirement on explanation. Those who take nomic expectability to be a necessary condition on explanation will agree with Hempel (1965, p. 337) when he tells us that a good explanation

shows that, given the particular circumstances and the laws in question, the occurrence of the phenomena was to be expected; and it is in this sense that the explanation enables us to understand why the phenomena occurred.

Deterministic chances, according to THE SUBJECTIVIST ACCOUNT, do precisely this for long-run frequencies. They show us that, if a €I coin is tossed I,000 times in a row, any reasonable credence distribution over the initial conditions of a true system of dynamical equations governing the outcomes of the sequence of coin tosses - were it informed about the relevant causal dynamics — would place around $95 \%$ of its credence in those sequences in which about half of the coin tosses land heads. And to show this is just to show that a frequency of about $\mathrm{I} / 2$ heads was to be expected, given the chances.

Few nowadays believe that nomic expectability is sufficient for explanation. At a minimum, we'll also need the right kind of causal relevance of explanans to explanandum. But the dynamics are causally relevant to the frequency of heads, analogously to the way that dynamics are causally relevant in equilibrium explanations. In both cases, dynamical information is known while the precise initial conditions are not; the only difference is that, in paradigm equilibrium explanations (why did the marble rest at the bottom of the bowl after being dropped in? Because the bottom of the bowl is the point of minimum potential energy), the explanandum is entailed by the explanans. Whereas, in explanations like $(€)$, the explanandum is merely made highly probable, in the sense that we ought to have a high credence in the explanandum, given the explanans. So if we accept a nomic expectability requirement on explanation, then deterministic chances-understood as THE SUBJECTIVIST ACCOUNT understands them—should be capable of explaining longrun frequencies.

You may think that nomic expectability is not necessary for explanation. You may think that all that's required for a proper explanation is that the explanans are causally relevant to the explanandum. In this case, you are likely also an egalitarian 
about probabilistic explanation, and you should be content to notice that the causal dynamics of a deterministic chance are causally relevant to the outcome of the sequence of coin tosses, both in the case of $(€)$ and in the case of $(\mathfrak{E})$.

According to The subjectivist account, the claim that the coin had a I/2 chance of landing heads is a conjunction of a claim about the world's causal dynamics and a normative claim about what is to be expected, given the world's causal dynamics. All parties should agree that the causal component of a deterministic chance claim is explanatorily relevant to the long-run frequency, and that normative facts about what is to be expected are not. What subjectivist elitists and egalitarians disagree about is whether, in order for an explanation to be good, the explanans have to make the explanandum likely. Subjectivist egalitarians think not. Subjectivist elitists think so. So, in order to show that $€$ is a good explanation, the subjectivist elitist will have to appeal to the normative component of a deterministic chance. They will say: the normative fact that a long-run frequency of about $\mathrm{I} / 2$ heads landings was to be expected is necessary for the causal dynamics to explain why the coin landed heads about $\mathrm{I} / 2$ of the time. (Although this normative fact does not explain why the coin landed heads about $\mathrm{I} / 2$ of the time.)

LOEWER and Albert object strongly to the idea that chances, understood along the lines of THE SUBJECTIVIST ACCOUNT, could explain observed frequencies in this way. Speaking about statistical mechanical probabilities, LoEwer (200I, p. 6II, 6I5) writes:

What could your ignorance of the initial state of the gas have to do with an explanation of its behavior?...it is hard to see how these [subjective] probabilities can be explanatory if they are unrelated to the actual microstate.

With respect to LoEwer's rhetorical question-how could a rational initial credence distribution explain the behavior of something out in the world?- the correct answer is: it couldn't, but THE SUBJECTIVIST ACCOUNT does not have to, and should not, say that it does. The rational initial credence distributions do not explain why the coin landed as it did. What explains the frequency of heads landings is just the causal dynamics. Nevertheless, we may think that a good explanation must show that the explanandum was to expected. If so, then the normative component of a deterministic chance will explain why deterministic chances are able to explain some frequencies (viz., the ones which were to be expected), and not others (viz., the ones which weren't to be expected). Of course, explaining why the dynamics provide a good explanation of the frequency of heads is not itself explaining the frequency of heads. Facts about rational initial credences do the former; but they do not do the latter.

Compare: on HempeL's Deductive-Nomological model of explanation, in order for an explanation to be good, the explanans must entail the explanandum. On this model, in order to show that an explanation is good, you must appeal to a logical fact about entailment. Is this logical fact explanatorily relevant to the 
explanandum? No. The explanans do all the explanatory work by themselves. It is in virtue of the logical fact that the explanans are able to explain the explanandum, but the logical fact is not to be included among the explanans. 55 Similarly, the subjectivist elitist should say: in order for an explanation of the long-run frequency of heads to be good, it must be that this frequency is to be expected, given the explanans. In order to show that the subjectivist's explanation of the longrun frequency of heads is good, they must appeal to a normative fact about what is to be expected. Is this normative fact explanatorily relevant to the explanandum? No. The causal dynamics do all the explanatory work by themselves. It is in virtue of the normative fact that the causal dynamics are able to explain the long-run frequency of heads, but the normative fact is not to be included among the explanans.

One thing that appears to bother ALBERT and LOEwER about explaining longrun frequencies in the way THE SUBJECTIVIST ACCOUNT does is that the chances don't entail that the frequencies will be close to the chances. For instance, LoEwER (200I, p. 6I5) worries that "even if the actual state were a very atypical state the standard probability distribution would be dictated by [THE SUBJECTIVIST ACCOUNT]." 56 This, however, shouldn't be an objection to any account of chance. The natural thought is that the chance of heads being $\mathrm{I} / 2$ doesn't entail that not every flip will land heads. Some Humeans are willing to bite the bullet and claim that it does, but even LEwIS admits that this consequence of his view is "certainly very peculiar". ${ }^{57}$ It seems to me, then, that it is a feature, and not a bug, of THE SUBJECTIVIST ACCOUNT that it allows the possibility of low-chance outcomes. ${ }^{58}$ If you're inclined to think that only explanans which entail the explanandum are genuinely explanatory, then so be it-but, in that case, you shouldn't expect that chances can explain long-run frequencies.

\section{Conclusion}

Deterministic chance is an odd sort of beast. It appears at once subjective and epistemic, a chimera of our ignorance, and objective and worldly, the kind of thing that depends upon and helps to explain features of the natural world. I believe that the subjectivist account of deterministic chance developed here puts us in a position to appreciate this dual nature. According to the subjectivist account, deterministic chances depend upon objective features of the causal dynamics of

35 Cf. Carroll (I895).

56 LOEWER is considering an account predicated on the principle of indifference, but his objections apply with equal force to THE SUBJECTIVIST ACCOUNT.

57 LEWIS (I994, p. 483)

58 Shouldn't we still want there to be some connection between chance and frequency? Of course we should. And there is: as the number of independent trials gets larger, so too does the chance that the frequency of an outcome is close to the chance of that outcome. This is the law of large numbers (stated roughly). It is the most that we should ever want an account of chance to say about the connection between frequency and chance; for it is the most that is true about the connection between chance and frequency. And THE SUBJECTIVIST ACCOUNT says it. 
the world. For instance, they are sensitive to the actual distribution of mass in the die, since the distribution of mass in the die makes a difference to the dynamics mapping initial conditions of the die roll to outcomes. So too do these chances depend upon epistemic facts predicated upon a certain kind of ignorance-though not necessarily our ignorance. They depend upon the fact that, in ignorance of the precise initial conditions of a deterministically chancy process, it is irrational to be very confident in any narrow range of possible initial conditions. While deterministic chance does not depend upon our ignorance, its usefulness does. Of course, were we to be aware of the precise initial conditions of a deterministically chancy process, along with the corresponding dynamics, we would perhaps have no need of deterministic chances; we could predict and explain without them. Recall the coin flipping machine constructed by Diaconis et al. (2007). With this machine, it does not matter that we don't know the precise physical conditions of the coin's toss; for we know something more: we know that the outcome is additionally governed by a dynamical equation which maps states in which the coin is set down heads up to states in which the coin lands heads up, and we know that the coin is set down heads up. This information is inadmissible with respect to the $\mathrm{I} / 2$ chance determined by the dynamical equation $\phi_{H}$ from $\$ 2.2$. When we have this information, the $\mathrm{I} / 2$ chance of heads remains, though it no longer has any normative purchase on us. We ought not use it to determine our confidence that the coin lands heads. Regularly, however, we lack information like this. In these cases, knowledge of deterministic chances becomes invaluable. This explains why deterministic chances are able to play the role of an epistemic expert. A deterministic chance is just the credence that it would be rational to have, were we to know more about the causal dynamics of the world than we in fact know. By analyzing frequency data, we may come to know something about what that credence is without thereby coming to know anything about the underlying dynamics which inform it—we are afforded a glimpse, if only a glimpse, of the opinions of a better informed, rational agent. 


\section{REFERENCES}

Abrams, Marshall. 20I2. "Mechanistic Probability." Synthese, vol. I87 (2): 343-375. [4], [17]

Albert, David Z. 2000. Time and Chance. Harvard University Press, Cambridge, Ma. [4], [17], [32], [33]

—. 2015. After Physics. Harvard University Press, Cambridge, MA. [4], [17]

Bartle, Robert G. 1966. The Elements of Integration and Lebesgue Measure. Wiley Classics Library Edition. John Wiley \& Sons, Inc., New York. [II]

Beisbart, Claus. 20I6. "A Humean Guide to Spielraum Probabilities." Journal for General Philosophy of Science, vol. 47: 189-216. [4], [17]

Bradley, Seamus. 2017. "Are objective chances compatible with determinism?" Philosophy Compass, vol. I2 (8). [2]

Butterfield, Jeremy. 20II. "Less is Different: Emergence and Reduction Reconciled." Foundations of Physics, vol. 4I (6): I065-II35. [8]

Carroll, Lewis. I895. "What the tortoise said to Achilles." Mind, vol. 4 (I4): 278-28o. [32]

Clark, Peter. 1987. "Determinism and Probability in Physics." Proceedings of the Aristotelian Society, Supplementary Volume, vol. 61: I85-210. [I]

De Finetti, Bruno. 1974. Theory of Probability, vol. I. Wiley, New York. [17]

Diaconis, Persi, Susan Holmes \& Richard Montgomery. 2007. "Dynamical Bias in the Coin Toss." SIAM Review, vol. 49 (2): 2II-235. [2], [9], [34]

Elga, AdAm. 20I3. "The Puzzle of the Unmarked Clock and the New Rational Reflection Principle.” Philosophical Studies, vol. I64 (I): I27-139. [22]

Engel, Eduardo. 1992. A Road to Randomness in Physical Systems, vol. 71 of Lecture notes in statistics. Springer-Verlag, Berlin. [5], [9]

Fine, KIT. 1975. "Vagueness, Truth, and Logic." Synthese, vol. 30: 265-300. [20]

Glynn, Luke. 20IO. "Deterministic Chance." The British Journal for the Philosophy of Science, vol. 6I (I): 5I-80. [I]

Greco, Daniel. 20I4. "A Puzzle About Epistemic Akrasia." Philosophical Studies, vol. I67: 20I-219. [23]

Hempel, Carl. 1965. Aspects of Scientific Explanation and Other Essays in the Philosophy of Science. Free Press, New York. [31], [32]

Hoefer, Carl. 2007. "The Third Way of Objective Probability: A Skeptic's Guide to Objective Chance." Mind, vol. 463: 549-596. [I]

Hopf, Eberhard. 1934. "On Causality, Statistics, and Probability." Journal of Mathematics and Physics, vol. 13: 5I-IO2. [6]

Horowitz, Sophie. 20I4. "Epistemic Akrasia.” Nô̂s, vol. 48 (4): 718-744. [23]

Ismael, Jenann. 2009. "Probability in Deterministic Physics." Journal of Philosophy, vol. IO6: 89-109. [I]

Joyce, James M. 20io. "A Defense of Imprecise Credences in Inference and Decision Making." Philosophical Perspectives, vol. 24 (I): 28I-323. [20] 
Keefe, Rosanna. 2000. Theories of Vagueness. Cambridge University Press, Cambridge, UK. [20]

Keller, Joseph B. 1986. "The Probability of Heads." The American Mathematical Monthly, vol. 93 (3): I9I-I97. [6], [8], [9]

Laplace, Marquis De. I8I4. A Philosophical Essay on Probabilities. Dover Publications, New York. Translated from the 6th French Edition by F. W. TruscatT and F. L. EMORY. [3]

Lasonen-Aarnio, Maria. 20i4. "Higher-Order Evidence and the Limits of Defeat." Philosophy and Phenomenological Research, vol. 88 (2): 314-345. [23]

-. 20I5. "New Rational Reflection and Internalism about Rationality." In Oxford Studies in Epistemology, vol. 5, I45-171. Oxford University Press, Oxford. [23]

—. forthcoming. "Enkrasia or Evidentialism? Learning to Love Mismatch.” Philosophical Studies. [23]

Levi, Isaac. 1974. “On Indeterminate Probabilities.” Journal of Philosophy, vol. 7I: 39I4I8. [20]

Lewis, David K. I980. "A Subjectivist's Guide to Objective Chance." In Studies in Inductive Logic and Probability, Richard C. JeFFreY, editor, vol. II, 263-293. University of California Press, Berkeley. [I], [4], [20], [24], [27]

-. 1986. "A Subjectivist's Guide to Objective Chance." In Philosophical Papers, vol. II. Oxford University Press, Oxford. [I], [2]

—. 1994. "Humean Supervenience Debugged." Mind, vol. I03 (4I2): 473-490. [27], [33]

Loewer, Barry. 200I. "Determinism and Chance." Studies in the History and Philosophy of Modern Physics, vol. 32 (4): 609-620. [I], [4], [17], [32], [33]

- 2004. "David Lewis's Humean Theory of Objective Chance." Philosophy of Science, vol. 7I: III5-II25. [4], [I7]

- 2007. "Counterfactuals and the Second Law." In Causation, Physics, and the Constitution of Reality: Russell's Republic Revisited, Huw Price \& Richard Corry, editors, chap. II, 293-326. Oxford University Press, Oxford. [4], [I7]

Myrvold, Wayne C. 20I2. "Deterministic Laws and Epistemic Chances." In Probability in Physics, Y. Ben-Menahem \& M. Hemmo, editors, 73-85. Springer-Verlag, Berlin. [4], [I8]

Poincaré, Henri. 1905. Science and Hypothesis, chap. in: The Calculus of Probabilities. The Walter Scott Publishing Company, New York. [4]

-. 1912. Calcul des probabilités. Gauthier-Villars, Paris, second edn. [4], [6]

Popper, Karl. 1982. Quantum Theory and the Schism in Physics. Rowman and Littlefield, New Jersey. [I]

Ramsey, Frank P. 193I. "Truth and Probability." In Foundations of Mathematics and other Logical Essays, R.B. Braithwaite, editor, chap. VII, I56-I98. Kegan, Paul, Trench, Trubner \& Co. Ltd., London. [I7]

Reichenbach, Hans. 1971. The Theory of Probability: An Inquiry into the Logical and Mathematical Foundations of the Calculus of Probabilities. University of California Press, Berkeley, second edn. [i6] 
Roberts, John T. 20i6. “The Range Conception of Probability and the Input Problem." Journal for General Philosophy of Science, vol. 47: 17I-I88. [4], [17]

Rosenthal, Јасов. 20Iо. "The Natural-range Conception of Probability." In Time, Chance, and Reduction: Philosophical Aspects of Statistical Mechanics, Gerhard ERnst $\&$ Andreas Hüttemann, editors, 7I-9I. Cambridge University Press, Cambridge. [4], [I7]

-. 20I2. "Probabilities as Ratios of Ranges in Initial-State Spaces." Journal of Logic, Language and Information, vol. 21: 217-236. [4], [12], [13], [I5], [17]

-. 2016. "Johannes con Kries's Range Conception, the Method of Arbitrary Functions and Related Modern Approaches to Probability." Journal for General Philosophy of Science, vol. 47: I5I-170. [4], [17]

Savage, Leonard J. 1954. The Foundations of Statistics. Dover Publications, New York, second edn. [17]

—. 1971. "Probability in Science: A Personalistic Account." In Logic, Methodology, and Philosophy of Science, Patrick Suppes, Leon Henkin, Athanase Joja \& Gr. C. MoisiL, editors, vol. 4, 417-428. North-Holland Publishing Company, Amsterdam; London. [4], [I7], [I8]

Schaffer, Jonathan. 2007. "Deterministic Chance?" The British Journal for the Philosophy of Science, vol. 58: II3-I4O. [I], [2]

Sober, Elliott. 20Io. "Evolutionary Theory and the Reality of Macro Probabilities." In The Place of Probability in Science, Ellery Eells \& James H. Fetzer, editors, I33-I6r. Springer, Dordrecht. [I]

Strevens, Michael. 2000. "Do Large Probabilities Explain Better?” Philosophy of Science, vol. 67: 366-390. [30]

- 2003. Bigger than Chaos: Understanding Complexity through Probability. Harvard University Press, Cambridge, MA. [4], [5], [8], [9], [II], [I2], [14], [17]

-. 20iI. "Probability Out Of Determinism." In Probability in Physics, Claus Beisbart \& Stephen Hartmann, editors, 339-364. Oxford University Press, Oxford. [I], [4], [8], [II], [I7]

—. 2013. Tychomancy. Harvard University Press, Cambridge, MA. [9], [ІІ]

Titelbaum, Michael G. 20I4. "Rationality's Fixed Point (Or: In Defense of Right Reason)." Oxford Studies in Epistemology, vol. 5. [23]

van Fraassen, Bas C. 1990. "Figures in a Probability Landscape." In Truth or Consequences: Essays in Honor of Nuel Belnap, J. Michael Dunn \& Anil Gupta, editors, 345-356. Kluwer Academic Publishers, Dordrecht. [20]

—. 2006. "Vague Expectation Value Loss." Philosophical Studies, vol. I27: 483-49I. [20]

von Kries, Johannes. i886. Principien der Wahrscheinlichkeitsrechnung, eine logische Untersuchung. Mohr, Freiburg im Breisgau. [4]

von Plato, Jan. 1982. "Probability and Determinism." Philosophy of Science, vol. 49 (I): 5I-66. [4]

-. 1983. "The Method of Arbitrary Functions." The British Journal for the Philosophy of Science, vol. 34 (I): 37-47. [5], [I7] 
Walley, Peter. I99I. Statistical Reasoning with Imprecise Probabilities. Chapman \& Hall, London. [20]

White, Roger. 2005. “Epistemic Permissiveness." Philosophical Perspectives, vol. I9: 445459. [19], [20]

—. 2009. "Evidential Symmetry and Mushy Credence." Oxford Studies in Epistemology, I6I-I86. [20]

Williamson, Tiмотну. 1994. Vagueness. Routledge, London. [19]

-. 2000. Knowledge and its Limits. Oxford University Press, Oxford. [22]

- 20II. "Improbable Knowing." In Evidentialism and its Discontents, T. DougherTy, editor. Oxford University Press, Oxford. [22]

—. 20I4. "Very Improbable Knowing." Erkenntnis, vol. 79 (5): 97I-999. [22]

Woodward, James. 2016. "The Problem of Variable Choice." Synthese, vol. 193 (4): IO47-IO72. [13]

Zabell, Sandy. 2016. "Johannes von Kries's Principien: A Brief Guide for the Perplexed." Journal for General Philosophy of Science, vol. 47: 13I-I5O. [4] 